\title{
Steps toward determination of the size and structure of the broad-line region in active galactic nuclei. II - an intensive study of NGC 5548 at optical wavelengths
}

\section{Citation}

Peterson, B. M., T. J. Balonek, E. S. Barker, J. Bechtold, R. Bertram, N. G. Bochkarev, M. J. Bolte, et al. 1991. "Steps Toward Determination of the Size and Structure of the Broad-Line Region in A large, international program of ground-based optical spectroscopy and photometry of the variable Seyfert 1 galaxy NGC 5548 undertaken in support of an IUE monitoring campaign is described. This contribution presents the data base and describes the methods used to correct for systematic differences between spectra from different sources. Optical continuum and $\mathrm{H}$-beta emission-line light curves are derived from the spectra. The behavior of the optical continuum is qualitatively the same as the behavior of the ultraviolet continuum. Cross-correlation of the ultraviolet and optical continuum measurements does not reveal any significant lag between them. The h-beta emission-line variations show the same basic pattern as seen in the continuum and ultraviolet emission lines, with $\mathrm{H}$-beta lagging behind the continuum by about 20 days. This is significantly larger than the about 10 day lag deduced for Lyalpha.A large, international program of ground-based optical spectroscopy and photometry of the variable Seyfert 1 galaxy NGC 5548 undertaken in support of an IUE monitoring campaign is described. This contribution presents the data base and describes the methods used to correct for systematic differences between spectra from different sources. Optical continuum and $\mathrm{H}$-beta emission-line light curves are derived from the spectra. The behavior of the optical continuum is qualitatively the same as the behavior of the ultraviolet continuum. Cross-correlation of the ultraviolet and optical continuum measurements does not reveal any significant lag between them. The h-beta emission-line variations show the same basic pattern as seen in the continuum and ultraviolet emission lines, with $\mathrm{H}$-beta lagging behind the continuum by about 20 days. This is significantly larger than the about 10 day lag deduced for Ly-alpha.Active Galactic Nuclei. II - an Intensive Study of NGC 5548 at Optical Wavelengths." The Astrophysical Journal 368 (February): 119. doi:10.1086/169675.

\section{Published Version}

doi:10.1086/169675

\section{Permanent link}




\section{Terms of Use}

This article was downloaded from Harvard University's DASH repository, and is made available under the terms and conditions applicable to Other Posted Material, as set forth at http:// nrs.harvard.edu/urn-3:HUL.InstRepos:dash.current.terms-of-use\#LAA

\section{Share Your Story}

The Harvard community has made this article openly available.

Please share how this access benefits you. Submit a story.

Accessibility 


\title{
STEPS TOWARD DETERMINATION OF THE SIZE AND STRUCTURE OF THE BROAD-LINE REGION IN ACTIVE GALACTIC NUCLEI. II. AN INTENSIVE STUDY OF NGC 5548 AT OPTICAL WAVELENGTHS
}

B. M. Peterson,${ }^{1,2}$ T. J. Balonek, ${ }^{3}$ E. S. Barker, ${ }^{4}$ J. Bechtold,${ }^{5}$ R. Bertram, ${ }^{1}$ N. G. Bochkarev, ${ }^{6}$ M. J. Bolte, ${ }^{7}$ D. Bond, ${ }^{7}$ T. A. Boroson, ${ }^{8,9}$ M. T. Carini, ${ }^{10}$ T. E. Carone, ${ }^{11}$ J. A. Christensen, ${ }^{3}$ S. D. Clements, ${ }^{12}$ A. L. Cochran, ${ }^{4}$ R. D. Cohen, ${ }^{13}$ D. Crampton, ${ }^{7}$ M. Dietrich, ${ }^{14}$ M. Elvis,${ }^{15}$ A. Ferguson, ${ }^{7}$ A. V. Filippenko, ${ }^{16}$ K. J. Fricke, ${ }^{14}$ C. M. Gaskell, ${ }^{17}$ J. P. Halpern, ${ }^{18,19}$ J. P. Huchra, ${ }^{15}$ J. B. Hutchings, ${ }^{7}$ W. Kollatschny, ${ }^{14}$ A. P. KoratKar, ${ }^{17}$ K. T. Korista ${ }^{1}$ J. H. KroliK, ${ }^{20}$ N. J. LAME, ${ }^{1}$ A. LAOR, ${ }^{21}$ R. J. LeACOCK, ${ }^{12}$ G. M. MACAlPine, ${ }^{17}$ M. A. MalKan, ${ }^{22}$ D. MAOZ, ${ }^{21}$ H. R. Miller, ${ }^{10}$ S. L. Morris,${ }^{8}$ H. Netzer, ${ }^{21}$ C. L. M. Oliveira,${ }^{23}$ J. Penfold,${ }^{24}$ M. V. Penston, ${ }^{25}$ E. PÉrez, ${ }^{26}$ R. W. Pogge, ${ }^{1,4}$ M. W. Richmond, ${ }^{16}$ W. Romanishin, ${ }^{27}$ E. I. Rosenblatt, ${ }^{22}$ L. SAdDlemyer, ${ }^{7}$ A. Sadun, ${ }^{28}$ S. R. SAWyer, ${ }^{4}$ J. C. Shields, ${ }^{16}$ A. I. Shapovalova, ${ }^{29}$

\section{A. G. Smith, ${ }^{12}$ H. A. Smith, ${ }^{30}$ P. S. Smith, ${ }^{5}$ W.-H. Sun, ${ }^{31,32}$ U. ThiEle, ${ }^{33}$}

T. J. Turner, ${ }^{34}$ S. VeilleuX, ${ }^{2}$ R. M. Wagner, ${ }^{1}$ R. J. Weymann, ${ }^{8}$

B. J. Wilkes, ${ }^{15}$ B. J. Wills, ${ }^{4}$ D. Wills, ${ }^{4}$ AND P. F. Younger ${ }^{7}$

Received 1990 June 29 ; accepted 1990 August 3

\begin{abstract}
We report on a large, international program of ground-based optical spectroscopy and photometry of the variable Seyfert 1 galaxy NGC 5548 undertaken in support of an IUE monitoring campaign described by Clavel and coworkers. In this contribution, we present the data base and describe the methods used to correct for systematic differences between spectra from different sources. Optical continuum and $\mathrm{H} \beta$ emission-line light curves are derived from the spectra. The behavior of the optical continuum is qualitatively the same as the behavior of the ultraviolet continuum. Cross-correlation of the ultraviolet and optical continuum measurements does not reveal any significant lag between them. The $\mathrm{H} \beta$ emission-line variations show the same basic pattern as seen in the continuum and ultraviolet emission lines, with $\mathrm{H} \beta$ lagging behind the continuum by $\sim 20$ days. This is significantly larger than the $\sim 10$ day lag deduced for Ly $\alpha$.
\end{abstract}

Subject headings: galaxies: individual (NGC 5548) - galaxies: Seyfert - spectrophotometry

${ }^{1}$ Department of Astronomy, The Ohio State University.

2 University of California Observatories/Lick Observatory, University of California, Santa Cruz.

${ }^{3}$ Department of Physics and Astronomy, Colgate University.

${ }^{4}$ McDonald Observatory and Department of Astronomy, University of Texas at Austin.

${ }^{5}$ Steward Observatory, University of Arizona.

${ }^{6}$ Sternberg State Astronomical Institute, University of Moscow.

${ }^{7}$ Dominion Astrophysical Observatory, National Research Council of Canada.

${ }^{8}$ Observatories of the Carnegie Institution of Washington.

${ }^{9}$ Kitt Peak National Observatory, National Optical Astronomy Observatories.

10 Department of Physics and Astronomy, Georgia State University.

${ }^{11}$ Lunar and Planetary Laboratory-West, University of Arizona.

12 Rosemary Hill Observatory, University of Florida.

13 Center for Astrophysics and Space Sciences, University of California, San Diego.

${ }^{14}$ Universitäts-Sternwarte Göttingen.

${ }^{15}$ Center for Astrophysics, Smithsonian Astrophysical Observatory.

${ }^{16}$ Department of Astronomy, University of California, Berkeley.

17 Department of Astronomy, University of Michigan.

${ }^{18}$ Department of Astronomy, Columbia University.

19 Visiting Astronomer, Kitt Peak National Observatory, National Optical Astronomy Observatories, which is operated by AURA, Inc., under a cooperative agreement with the National Science Foundation.

${ }^{20}$ Department of Physics and Astronomy, Johns Hopkins University.

${ }^{21}$ School of Physics and Astronomy and the Wise Observatory, Tel-Aviv University.

${ }^{22}$ Department of Astronomy, University of California, Los Angeles.

${ }^{23}$ Department of Astronomy, University of British Columbia.

${ }^{24}$ Department of Mathematics, Physics, and Engineering, Mount Royal College.

${ }^{25}$ Royal Greenwich Observatory.

${ }^{26}$ Instituto de Astrofisica de Canarias.

27 Department of Physics and Astronomy, University of Oklahoma.

${ }^{28}$ Department of Physics and Astronomy, Agnes Scott College.

${ }^{29}$ Special Astrophysical Observatory, USSR Academy of Sciences.

${ }^{30}$ Department of Astronomy, Michigan State University.

${ }^{31}$ Laboratory for Astronomy and Solar Physics, Goddard Space Flight Center.

${ }^{31}$ Institute of Physics and Astronomy, National Central University

${ }^{33}$ Max-Planck-Institut für Astronomie, Heidelberg.

${ }^{34}$ Laboratory for High Energy Astrophysics, Goddard Space Flight Center. 


\section{INTRODUCTION}

Variability of the continuum and emission lines in active galactic nuclei (AGNs) is a well-established phenomenon (see Peterson 1988 for a review). The observed variations in the broad emission lines are apparently strongly correlated with variations in the ultraviolet and optical continua. This single fact suggests that it may be possible to determine the size and structure of the broad-line region (BLR) from the detailed response of the emission lines to the changes in the continuum flux, although it is certainly clear that there are many pitfalls that one may encounter in actual practice. Nevertheless, the possibility of extracting such fundamental information about these spatially unresolved regions has led to considerable efforts to monitor the variable spectra of AGNs. Only recently has the observational problem become sufficiently well defined that it is possible to obtain reliable results (see Netzer 1989).

In order to resolve temporally AGN continuum and emission-line behavior sufficiently well that it is possible to infer reliable structural information about the emission-line regions, an enormous amount of data is required, at least by the standards of faint-object astronomy. Recognition of this led to a large international effort to observe the Seyfert 1 galaxy NGC 5548 with the International Ultraviolet Explorer (IUE) every 4 days for an 8 month period. The results of this experiment are reported by Clavel et al. (1991, hereafter Paper I). It was also recognized that a concurrent ground-based program would enhance the scientific return on this considerable investment of $I U E$ time (1) by extending the continuum coverage through the optical and IR, (2) by including the important Balmer series emission lines in the study, and (3) by providing both velocity resolution and signal-to-noise ratios unattainable with $I U E$. Moreover, it might be possible to achieve even better temporal resolution than planned with $I U E$, at least during limited intervals. Weather considerations made cooperation among observatories imperative to achieve reasonable temporal resolution and minimize gaps in the temporal coverage. The principal disadvantage of this approach is that the data are not extremely homogeneous or even close to regularly sampled. While this certainly introduces difficulties, these have been found to be surmountable.

In this contribution we present the ground-based optical data on NGC 5548 obtained during the period 1988 December-1989 October when this galaxy was intensely studied with IUE, as described in Paper I. These data, together with the ultraviolet spectra of Paper I, constitute a unique large data base for study of AGN spectral variability. In this paper we focus our attention on the primary scientific goal of this project, namely, determination of the time scale for the response of the $\mathrm{H} \beta$ emission line to continuum variations. Further analysis of these data will appear in future papers.

We present the optical spectroscopy and photometry obtained in this study in $\S$ II. In $\S$ III we discuss how we have constructed a homogeneous data base from these observations to produce light curves for the optical continuum and the $\mathrm{H} \beta$ emission line. We perform some preliminary time-series analysis in $\S \mathrm{IV}$, and compare the results obtained here with the results of Paper I. Our conclusions are summarized in $\S \mathrm{V}$.

\section{OBSERVATIONS}

a) Optical Spectroscopy

Optical spectra of NGC 5548 were obtained at many observatories as part of this campaign. A complete log of spectro- scopic observations appears in Table 1. The UT date and Julian Date of each observation are given in columns (1) and (2), respectively. The column (3) entry indicates the observatory and instrument which obtained the spectrum. The projected spectrograph entrance aperture, in arcseconds, is given in column (4). For rectangular apertures, the first dimension is the slit width in the dispersion direction, and the second dimension is the slit length in the cross-dispersion direction; in the case of two-dimensional detectors (CCDs and IPCS), the second entry is the "extraction window" used. The slit position angle is given in column (5), measured eastward from north; the crossdispersion direction runs north-south for a position angle $0^{\circ}$. An estimate of the seeing is given in column (6), except for the case of the Wise Observatory data, which were obtained through such large apertures that the seeing is irrelevant. The nominal spectral resolution is given in column (7), and column (8) contains the approximate wavelength range covered by the data. Finally, to aid future investigators who will make use of these data, column (9) gives a unique identifier by which the spectrum is known to the IRAF reduction system, and which is contained in the FITS file header. The first two characters (" $n 5$ ") in this name identify the galaxy as NGC 5548, and the next four characters (e.g., “7509") contain the four least significant figures in the Julian Date, as in column (2). The next character gives the observatory code, as in column (3). When necessary, an additional arbitrary character is added to eliminate any remaining ambiguity.

\section{b) Photometry}

A program of photographic photometry was carried out with the $0.76 \mathrm{~m}$ telescope of the Rosemary Hill Observatory in Bronson, Florida. Photographs were obtained in three colors: $U$ (Kodak 103a-O + UG-2), B (Kodak 103a-O + GG-385), and $V$ (Kodak 103a-D + GG-495). All plates were hypersensitized, and the exposure lengths were in each case 2 minutes or less. The galaxy and comparison star measurements were made with a Cuffey Iris Astrophotometer. To suppress the starlight contribution from the host galaxy, a fixed iris corresponding to $7^{\prime \prime}$ in diameter was used for all measurements. The measurements were calibrated by using four nearby comparison stars (Penston, Penston, and Sandage 1971). The results obtained in this program are summarized in Table 2.

A program of $U B V R$ photoelectric photometry was also carried out with the $0.61 \mathrm{~m}$ Table Mountain telescope, and the results are given in Table 3 . Note that the small differences between the first two sets of measurements, which are only one night apart, are undoubtedly due to the different aperture sizes used.

Broad-band photometric measurements were also obtained with a Photometrics liquid nitrogen-cooled CCD system with a Thomson 7882 chip on the $0.4 \mathrm{~m}$ telescope of Foggy Bottom Observatory of Colgate University. The combination of the CCD response and the blue (Mould) filter (kindly loaned by Kitt Peak National Observatory) approximates Johnson $B$. The integrated magnitudes inside a circular aperture of diameter $17^{\prime \prime} .5$, centered on the nucleus of the galaxy, are given in Table 4. Star 1 from Penston, Penston, and Sandage (1971) was used as a comparison star.

\section{ANALYSIS OF THE DATA}

While Table 1 contains a complete $\log$ of all spectra obtained in this program, we will at this time confine our attention only to those data which cover the $\mathrm{H} \beta$ spectral 
TABLE 1

Log of SPeCtroscopic Observations

\begin{tabular}{|c|c|c|c|c|c|c|c|c|}
\hline \multirow{2}{*}{ 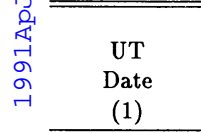 } & \multirow{2}{*}{$\begin{array}{c}\text { Julian Date } \\
(2440000+) \\
(2)\end{array}$} & \multirow[b]{2}{*}{$\begin{array}{c}\text { Code } \\
(3)\end{array}$} & \multicolumn{2}{|c|}{ Aperture } & \multirow{2}{*}{$\begin{array}{l}\text { Seeing } \\
(") \\
(6)\end{array}$} & \multirow{2}{*}{$\begin{array}{l}\text { Res. } \\
(\AA) \\
(7)\end{array}$} & \multirow{2}{*}{$\begin{array}{c}\text { Range } \\
(\AA) \\
(8)\end{array}$} & \multirow{2}{*}{$\begin{array}{l}\text { IRAF } \\
\text { file } \\
(9)\end{array}$} \\
\hline & & & $\begin{array}{l}\text { Size } \\
(4)\end{array}$ & $\begin{array}{c}\text { P.A. } \\
(5)\end{array}$ & & & & \\
\hline 1988 Dec 14 & 7509 & $\mathrm{H}$ & $4.0 \times 10.0$ & 120. & 3 & 5 & $4290-5100$ & n57530ha \\
\hline 1988 Dec 14 & 7509 & $\mathrm{H}$ & $15.0 \times 10.0$ & 120. & 3 & 10 & $4290-5100$ & n57530hb \\
\hline 1988 Dec 14 & 7509 & $\mathrm{H}$ & $4.0 \times 10.0$ & 120. & 3 & 20 & $3100-6280$ & n57530hc \\
\hline $1988 \operatorname{Dec} 17$ & 7512 & M & $2.4 \times 10.0$ & 0. & 1.5 & 2 & $4750-5200$ & n57512ma \\
\hline 1988 Dec 17 & 7512 & M & $2.4 \times 10.0$ & 0. & 1.5 & 2 & $6230-6800$ & $\mathrm{n} 57512 \mathrm{mb}$ \\
\hline 1988 Dec 22 & 7517 & A & $5.0 \times 7.6$ & 90. & $4-5$ & 9 & $4470-5620$ & $\mathrm{n} 57517 \mathrm{a}$ \\
\hline 1988 Dec 29 & 7524 & I & $4.5 \times 27.2$ & 90. & 2 & 14 & $3200-6050$ & $\mathrm{n} 57524 \mathrm{ia}$ \\
\hline 1988 Dec 29 & 7524 & I & $4.5 \times 27.2$ & 90. & 2 & 14 & $5800-8400$ & n57524ib \\
\hline $1988 \operatorname{Dec} 29$ & 7524 & I & $4.5 \times 27.2$ & 90. & 2 & 4 & $4600-5350$ & n57524ic \\
\hline 1988 Dec 30 & 7525 & I & $4.5 \times 27.2$ & 90. & $3-4$ & 14 & $3230-6100$ & $\mathrm{n} 57525 \mathrm{ia}$ \\
\hline 1988 Dec 30 & 7525 & I & $4.5 \times 27.2$ & 90. & $3-4$ & 14 & $5800-8400$ & n57525ib \\
\hline 1989 Jan 1 & 7528 & $\mathrm{~F}$ & $3.2 \times 6.4$ & 90. & 1 & 5 & $4540-7050$ & $\mathrm{n} 57528 \mathrm{f}$ \\
\hline 1989 Jan 4 & 7530 & M & $1.2 \times 10.0$ & 90. & 2 & 4 & $6200-7000$ & $\mathrm{n} 57530 \mathrm{~m}$ \\
\hline 1989 Jan 4 & 7530 & $\mathrm{H}$ & $4.0 \times 10.0$ & 131. & 2 & 5 & $4350-5150$ & n57530ha \\
\hline 1989 Jan 4 & 7530 & $\mathrm{H}$ & $15.0 \times 10.0$ & 131. & 2 & 10 & $4350-5150$ & $\mathrm{n} 57530 \mathrm{hb}$ \\
\hline 1989 Jan 6 & 7532 & M & $1.2 \times 10.0$ & 0. & 1.5 & 4 & $6200-7000$ & $\mathrm{n} 57532 \mathrm{~m}$ \\
\hline 1989 Jan 6 & 7533 & $\mathrm{~F}$ & $3.2 \times 6.4$ & 90. & $1-2$ & 5 & $4540-7050$ & n57533f \\
\hline 1989 Jan 8 & 7534 & A & $5.0 \times 7.6$ & 90. & $2-3$ & 9 & $4610-5580$ & $\mathrm{n} 57534 \mathrm{a}$ \\
\hline 1989 Jan 9 & 7535 & M & $1.5 \times 10.0$ & 0. & 1.0 & 3 & $6200-7000$ & $\mathrm{n} 57535 \mathrm{~m}$ \\
\hline 1989 Jan 9 & 7535 & A & $5.0 \times 7.6$ & 90. & $2-3$ & 9 & $4560-5710$ & $\mathrm{n} 57535 \mathrm{a}$ \\
\hline 1989 Jan 12 & 7539 & $\mathrm{~F}$ & $3.2 \times 6.4$ & 90. & $1-2$ & 5 & $4540-7050$ & $\mathrm{n} 57539 \mathrm{f}$ \\
\hline 1989 Jan 13 & 7539 & M & $2.1 \times 10.0$ & 0. & 2 & 10 & $4000-6900$ & $\mathrm{n} 57539 \mathrm{~m}$ \\
\hline 1989 Jan 16 & 7543 & $\mathrm{~F}$ & $3.2 \times 6.4$ & 90. & 1 & 5 & $4540-7050$ & $\mathrm{n} 57543 \mathrm{f}$ \\
\hline 1989 Jan 20 & 7546 & M & $2.1 \times 10.0$ & 0. & $3-4$ & 10 & $4000-6900$ & $\mathrm{n} 57546 \mathrm{~m}$ \\
\hline 1989 Jan 23 & 7549 & M & $2.0 \times 10.0$ & 0. & 2.5 & 5 & $3800-5500$ & $\mathrm{n} 57549 \mathrm{~m}$ \\
\hline 1989 Jan 30 & 7556 & $\mathrm{H}$ & $2.1 \times 7.9$ & 90. & 2 & 7 & $4200-5800$ & n57556ha \\
\hline 1989 Jan 30 & 7556 & $\mathrm{H}$ & $7.0 \times 7.9$ & 90. & 2 & 18 & $4200-5800$ & $\mathrm{n} 57556 \mathrm{hb}$ \\
\hline 1989 Feb 3 & 7560 & M & $1.8 \times 10.0$ & 0. & 3 & 3 & $4720-5670$ & $\mathrm{n} 57560 \mathrm{~m}$ \\
\hline 1989 Feb 3 & 7560 & A & $5.0 \times 7.6$ & 90. & $3-4$ & 9 & $4500-5620$ & $\mathrm{n} 57560 \mathrm{a}$ \\
\hline $\begin{array}{l}1989 \text { Feb } 3 \\
1989 \text { Feb } 6\end{array}$ & $\begin{array}{l}7561 \\
7564\end{array}$ & $\begin{array}{l}\mathrm{E} \\
\mathrm{E}\end{array}$ & $\begin{array}{l}5.0 \times 9.6 \\
5.0 \times 11.8\end{array}$ & $\begin{array}{l}90 . \\
90 .\end{array}$ & $\begin{array}{l}5 \\
3\end{array}$ & $\begin{array}{l}5 \\
3\end{array}$ & $\begin{array}{l}4440-5400 \\
4440-5400\end{array}$ & $\begin{array}{c}\mathrm{n} 57561 \mathrm{e} \\
\mathrm{n} 57564 \mathrm{e}\end{array}$ \\
\hline $1989 \mathrm{Feb} 7$ & 7565 & $\mathrm{E}$ & $5.0 \times 8.6$ & 90. & 2 & 3 & $4440-5400$ & $\mathrm{n} 57565 \mathrm{e}$ \\
\hline 1989 Feb 9 & 7567 & $\mathrm{E}$ & $5.0 \times 9.8$ & 90. & 3 & 5 & $4450-5400$ & $\mathrm{n} 57567 \mathrm{e}$ \\
\hline $1989 \mathrm{Feb} 10$ & 7568 & $\mathrm{E}$ & $5.0 \times 12.6$ & 90. & 3 & 5 & $4400-5360$ & $\mathrm{n} 57568 \mathrm{e}$ \\
\hline 1989 Feb 12 & 7570 & $\mathrm{~F}$ & $3.2 \times 6.4$ & 90. & $1-2$ & 5 & $4540-7050$ & n57570f \\
\hline 1989 Feb 13 & 7571 & $\mathrm{~F}$ & $3.2 \times 6.4$ & 90. & $1-2$ & 5 & $4540-7050$ & n57571f \\
\hline 1989 Feb 14 & 7572 & $\mathrm{E}$ & $5.0 \times 8.8$ & 90. & 2 & 5 & $4430-5400$ & $\mathrm{n} 57572 \mathrm{e}$ \\
\hline 1989 Feb 15 & 7572 & $M$ & $1.2 \times 10.0$ & 0. & 2 & 2 & $6100-6980$ & $\mathrm{n} 57572 \mathrm{~m}$ \\
\hline $1989 \mathrm{Feb} 16$ & 7573 & B & $20.0 \times 28.0$ & 0. & & 10 & $4670-7020$ & $\mathrm{n} 57573 \mathrm{~b}$ \\
\hline 1989 Feb 16 & 7573 & M & $1.2 \times 10.0$ & 0. & 1.5 & 2 & $4600-5480$ & $\mathrm{n} 57573 \mathrm{~m}$ \\
\hline 1989 Feb 16 & 7573 & $\mathrm{~A}$ & $5.0 \times 7.6$ & 90. & 2 & 9 & $4450-5600$ & $\mathrm{n} 57573 \mathrm{a}$ \\
\hline 1989 Feb 17 & 7574 & M & $3.0 \times 10.0$ & 0. & 1 & 9 & $4000-7010$ & $\mathrm{n} 57574 \mathrm{~m}$ \\
\hline 1989 Feb 17 & 7574 & I & $4.5 \times 27.2$ & 90. & 2 & 14 & $3200-6100$ & n57574ia \\
\hline 1989 Feb 17 & 7574 & I & $4.5 \times 27.2$ & 90. & 2 & 14 & $5800-7500$ & n57574ib \\
\hline 1989 Feb 17 & 7574 & I & $4.5 \times 27.2$ & 90. & 2 & 4 & $4550-5300$ & $\mathrm{n} 57574 \mathrm{ic}$ \\
\hline 1989 Feb 18 & 7575 & M & $2.0 \times 10.0$ & 0. & 1.5 & 2 & $4400-5250$ & n57575ma \\
\hline $1989 \mathrm{Feb} 18$ & 7575 & M & $2.0 \times 10.0$ & 0. & 1.5 & 2 & $6100-7200$ & $\mathrm{n} 57575 \mathrm{mb}$ \\
\hline 1989 Feb 19 & 7576 & M & $2.0 \times 10.0$ & 0. & 2 & 4 & $3840-5420$ & $\mathrm{n} 57576 \mathrm{~m}$ \\
\hline 1989 Feb 25 & 7582 & $\mathrm{~A}$ & $5.0 \times 7.6$ & 90. & 4 & 9 & $4590-5710$ & $\mathrm{n} 57582 \mathrm{a}$ \\
\hline 1989 Feb 26 & 7583 & $\mathrm{~N}$ & $4.6 \times 19.2$ & 0. & 2 & 16 & $4180-8660$ & n57583n \\
\hline 1989 Feb 27 & 7584 & B & $20.0 \times 28.0$ & 0. & & 10 & $4600-7020$ & n $57584 \mathrm{~b}$ \\
\hline 1989 Mar 2 & 7587 & $M$ & $2.0 \times 10.0$ & 90. & 2 & 11 & $4230-7350$ & $\mathrm{n} 57587 \mathrm{~m}$ \\
\hline 1989 Mar 2 & 7587 & $\mathbf{F}$ & $3.2 \times 6.4$ & 90. & $1-2$ & 5 & $4510-6950$ & n57587f \\
\hline 1989 Mar 4 & 7589 & M & $2.0 \times 10.0$ & 0. & 1.5 & 11 & $3830-7170$ & $\mathrm{n} 57589 \mathrm{~m}$ \\
\hline 1989 Mar 4 & 7589 & $\mathrm{~A}$ & $5.0 \times 7.6$ & 90. & 5 & 9 & $4460-5560$ & $\mathrm{n} 57589 \mathrm{a}$ \\
\hline 1989 Mar 5 & 7590 & $\mathrm{~L}$ & 2.0 round & - & 3 & 3 & $4240-5060$ & n575901 \\
\hline 1989 Mar 5 & 7590 & $\mathrm{~A}$ & $5.0 \times 7.6$ & 90. & $4-5$ & 9 & $4430-5550$ & n57590a \\
\hline 1989 Mar 5 & 7591 & $\mathbf{F}$ & $3.2 \times 6.4$ & 90. & $2-3$ & 5 & $4510-6950$ & n57591f \\
\hline 1989 Mar 6 & 7592 & $\mathrm{~F}$ & $3.2 \times 6.4$ & 90. & $2-3$ & 5 & $4510-6950$ & n57592f \\
\hline 1989 Mar 7 & 7592 & M & $2.0 \times 10.0$ & 90. & 1 & 11 & $3830-7160$ & $\mathrm{n} 57592 \mathrm{~m}$ \\
\hline 1989 Mar 7 & 7592 & $\mathrm{~J}$ & $7.0 \times 7.2$ & 0. & 2 & 11 & $3300-6000$ & n57592j \\
\hline 1989 Mar 7 & 7593 & $\mathrm{~F}$ & $3.2 \times 6.4$ & 90. & 1 & 5 & $4510-6950$ & n57593fa \\
\hline 1989 Mar 8 & 7593 & $\mathrm{~J}$ & $7.0 \times 7.2$ & 0. & 2 & 11 & $4500-7190$ & $\mathrm{n} 57593 \mathrm{j}$ \\
\hline $1989 \operatorname{Mar} 8$ & 7593 & $\mathrm{~F}$ & $3.2 \times 6.4$ & 90. & 1 & 5 & $4510-6950$ & n57593fb \\
\hline 1989 Mar 9 & 7594 & M & $2.6 \times 10.0$ & 0. & 2 & 2 & $4630-5500$ & $\mathrm{n} 57594 \mathrm{~m}$ \\
\hline 1989 Mar 12 & 7597 & M & $2.6 \times 10.0$ & 0. & 2 & 2 & $4630-5500$ & $\mathrm{n} 57597 \mathrm{~m}$ \\
\hline 1989 Mar 13 & 7598 & $\mathrm{~F}$ & $3.2 \times 6.4$ & 90. & 1 & 5 & $4510-6950$ & n57598f \\
\hline 1989 Mar 14 & 7599 & $\mathrm{~F}$ & $3.2 \times 6.4$ & 90. & 1 & 5 & $4510-6950$ & n57599f \\
\hline 1989 Mar 14 & 7599 & $\mathrm{~K}$ & $1.7 \times 3.0$ & 0. & 1.5 & 4 & $4230-5500$ & n57599ka \\
\hline 1989 Mar 14 & 7599 & $\mathrm{~K}$ & $1.7 \times 3.0$ & 0. & 1.5 & 10 & $4550-7400$ & n57599kb \\
\hline 1989 Mar 15 & 7600 & $\mathrm{~A}$ & $5.0 \times 7.6$ & 90. & $2-3$ & 9 & $4470-5600$ & $\mathrm{n} 57600 \mathrm{a}$ \\
\hline
\end{tabular}


TABLE 1-Continued

\begin{tabular}{|c|c|c|c|c|c|c|c|c|}
\hline \multirow{2}{*}{$\begin{array}{c}\text { UT } \\
\text { Date } \\
(1)\end{array}$} & \multirow{2}{*}{$\begin{array}{c}\text { Julian Date } \\
(2440000+) \\
(2)\end{array}$} & \multirow[b]{2}{*}{$\begin{array}{c}\text { Code } \\
\text { (3) }\end{array}$} & \multicolumn{2}{|c|}{ Aperture } & \multirow{2}{*}{$\begin{array}{l}\text { Seeing } \\
(") \\
(6)\end{array}$} & \multirow{2}{*}{$\begin{array}{l}\text { Res. } \\
(\AA) \\
(7)\end{array}$} & \multirow{2}{*}{$\begin{array}{c}\text { Range } \\
(\AA) \\
(8)\end{array}$} & \multirow{2}{*}{$\begin{array}{c}\text { IRAF } \\
\text { file } \\
(9)\end{array}$} \\
\hline & & & $\begin{array}{c}\text { Size } \\
(4)\end{array}$ & $\begin{array}{l}\text { P.A. } \\
(5)\end{array}$ & & & & \\
\hline 1989 Mar 15 & 7600 & $\mathrm{~K}$ & $6.7 \times 3.0$ & 0. & 1.5 & 14 & $4520-7500$ & $\mathrm{n} 57600 \mathrm{k}$ \\
\hline 1989 Mar 16 & 7601 & M & $2.0 \times 10.0$ & 0. & 1.5 & 2 & $4630-5500$ & $\mathrm{n} 57601 \mathrm{~m}$ \\
\hline 1989 Mar 16 & 7601 & $\mathrm{~K}$ & $1.7 \times 3.0$ & 0. & 1.5 & 10 & $4520-7500$ & $\mathrm{n} 57601 \mathrm{k}$ \\
\hline $1989 \mathrm{Mar} 21$ & 7606 & A & $5.0 \times 7.6$ & 90. & 2 & 9 & $4450-5500$ & $\mathrm{n} 57606 \mathrm{a}$ \\
\hline 1989 Mar 21 & 7606 & M & $2.0 \times 10.0$ & 0. & 4 & 2 & $4620-5500$ & $\mathrm{n} 57606 \mathrm{~m}$ \\
\hline 1989 Mar 28 & 7613 & I & $4.5 \times 27.2$ & 90. & $3-4$ & 14 & $3200-5860$ & n57613ia \\
\hline 1989 Mar 28 & 7613 & I & $4.5 \times 27.2$ & 90. & $3-4$ & 14 & $5550-7500$ & n57613ib \\
\hline 1989 Mar 28 & 7613 & I & $4.5 \times 27.2$ & 90. & $3-4$ & 4 & $4400-5140$ & n57613ic \\
\hline 1989 Mar 29 & 7614 & H & $4.0 \times 10.0$ & 60. & 3 & 20 & $3120-9180$ & n57614h \\
\hline 1989 Mar 30 & 7615 & F & $3.2 \times 6.4$ & 90. & 1 & 5 & $4470-6960$ & n57615f \\
\hline $1989 \mathrm{Mar} 31$ & 7616 & D & $8.0 \times 4.0$ & 66.9 & 1.5 & 13 & $3370-9660$ & n57616da \\
\hline 1989 Mar 31 & 7616 & D & $1.0 \times 4.0$ & 66.9 & 1.5 & 6 & $3370-9660$ & $\mathrm{n} 57616 \mathrm{db}$ \\
\hline 1989 Apr 1 & 7617 & F & $3.2 \times 6.4$ & 90. & 1 & 5 & $4520-7050$ & n57617f \\
\hline 1989 Apr 2 & 7618 & $\mathrm{~F}$ & $3.2 \times 6.4$ & 90. & 3 & 5 & $4520-7050$ & $\mathrm{n} 57618 \mathrm{f}$ \\
\hline 1989 Apr 2 & 7618 & A & $5.0 \times 7.6$ & 90. & 1.5 & 9 & $4470-5580$ & $\mathrm{n} 57618 \mathrm{a}$ \\
\hline $1989 \mathrm{Apr} 4$ & 7620 & $\mathrm{Q}$ & $4.0 \times 4.0$ & 90. & $1-2$ & 9 & $3520-6560$ & $\mathrm{n} 57620 \mathrm{q}$ \\
\hline 1989 Аpr 4 & 7620 & $\mathbf{F}$ & $3.2 \times 6.4$ & 90. & 2 & 5 & $4520-7050$ & $\mathrm{n} 57620 \mathrm{f}$ \\
\hline 1989 Apr 4 & 7621 & $\mathrm{H}$ & $2.1 \times 8.1$ & 90. & $3-4$ & 7 & $4000-6500$ & $\mathrm{n} 57621 \mathrm{~h}$ \\
\hline 1989 Apr 5 & 7621 & $\mathrm{~F}$ & $3.2 \times 6.4$ & 90. & 2 & 5 & $4550-7050$ & n57621f \\
\hline $1989 \mathrm{Apr} 6$ & 7623 & $\mathrm{Q}$ & $4.0 \times 4.0$ & 90. & $4-5$ & 9 & $3520-6560$ & $\mathrm{n} 57622 \mathrm{q}$ \\
\hline 1989 Apr 7 & 7623 & $\mathrm{~F}$ & $3.2 \times 6.4$ & 90. & 2 & 5 & $4510-7050$ & $\mathrm{n} 57623 \mathrm{f}$ \\
\hline $1989 \mathrm{Apr} 7$ & 7623 & $\mathrm{~K}$ & $2.4 \times 3.0$ & 0. & 1.5 & 10 & $4760-7540$ & n57623k \\
\hline 1989 Apr 8 & 7624 & $\mathrm{~F}$ & $3.2 \times 6.4$ & 90. & 2 & 5 & $4510-7050$ & $\mathrm{n} 57624 \mathrm{f}$ \\
\hline 1989 Apr 8 & 7624 & $\mathrm{~K}$ & $2.4 \times 3.0$ & 0. & 1.5 & 10 & $4750-7560$ & $\mathrm{n} 57624 \mathrm{k}$ \\
\hline 1989 Арг 10 & 7626 & $\mathrm{~F}$ & $3.2 \times 6.4$ & 90. & 2 & 5 & $4510-7050$ & n57626f \\
\hline 1989 Apr 10 & 7626 & $\mathrm{~K}$ & $2.4 \times 3.0$ & 0. & 1.5 & 10 & $4760-7550$ & $\mathrm{n} 57626 \mathrm{k}$ \\
\hline 1989 Арг 11 & 7627 & $\mathrm{~F}$ & $3.2 \times 6.4$ & 90. & 2 & 5 & $4510-7050$ & $\mathrm{n} 57627 \mathrm{f}$ \\
\hline 1989 Apr 11 & 7627 & A & $5.0 \times 7.6$ & 90. & $4-5$ & 9 & $4520-5640$ & $\mathrm{n} 57627 \mathrm{a}$ \\
\hline 1989 Арr 12 & 7628 & $\mathrm{H}$ & $2.1 \times 7.9$ & 90. & 2.5 & 7 & $4200-5820$ & n57628ha \\
\hline 1989 Apr 12 & 7628 & $\mathrm{H}$ & $7.0 \times 7.9$ & 90. & 2.5 & 18 & $4200-5820$ & $\mathrm{n} 57628 \mathrm{hb}$ \\
\hline 1989 Apr 13 & 7629 & $\mathrm{E}$ & $5.0 \times 11.2$ & 90. & 3 & 4 & $4620-5270$ & $\mathrm{n} 57629 \mathrm{e}$ \\
\hline 1989 Арг 15 & 7631 & $P$ & $8.0 \times 9.0$ & 90. & 2 & 20 & $4400-7100$ & $\mathrm{n} 57631 \mathrm{p}$ \\
\hline 1989 Apr 15 & 7631 & $\mathrm{~N}$ & $4.6 \times 19.2$ & 0. & 3 & 18 & $4300-8660$ & $\mathrm{n} 57631 \mathrm{n}$ \\
\hline 1989 Apr 16 & 7632 & $P$ & $8.0 \times 9.0$ & 90. & $2-3$ & 20 & $4400-7100$ & $\mathrm{n} 57632 \mathrm{p}$ \\
\hline 1989 Арг 26 & 7642 & A & $5.0 \times 7.6$ & 90. & $4-5$ & 9 & $4490-5620$ & n57642a \\
\hline 1989 Apr 27 & 7643 & $\mathrm{~F}$ & $3.2 \times 6.4$ & 90. & 2 & 5 & $4550-7050$ & $\mathrm{n} 57643 \mathrm{f}$ \\
\hline 1989 Арг 27 & 7643 & $\mathrm{H}$ & $4.0 \times 10.0$ & 60. & 2 & 20 & $3110-9180$ & $\mathrm{n} 57643 \mathrm{~h}$ \\
\hline $1989 \mathrm{Apr} 27$ & 7644 & M & $2.1 \times 10.0$ & 0. & 5 & 2 & $4750-5310$ & $\mathrm{n} 57644 \mathrm{~m}$ \\
\hline 1989 Apr 28 & 7644 & B & $20.0 \times 28.0$ & 0. & & 10 & $4620-7010$ & $\mathrm{n} 57644 \mathrm{~b}$ \\
\hline $1989 \mathrm{Apr} 28$ & 7644 & $\mathrm{~F}$ & $3.2 \times 6.4$ & 90. & 2 & 5 & $4540-7050$ & $\mathrm{n} 57644 \mathrm{f}$ \\
\hline 1989 Apr 29 & 7645 & I & $4.5 \times 27.2$ & 60. & $3-4$ & 14 & $3200-6000$ & n57645ia \\
\hline 1989 Арг 29 & 7645 & I & $4.5 \times 27.2$ & 60. & $3-4$ & 14 & $5500-7500$ & n57645ib \\
\hline 1989 Арг 29 & 7645 & I & $4.5 \times 27.2$ & 60. & $3-4$ & 4 & $4370-5150$ & $\mathrm{n} 57645 \mathrm{ic}$ \\
\hline 1989 May 1 & 7648 & M & $2.1 \times 10.0$ & 0. & 3 & 2 & $4750-5320$ & $\mathrm{n} 57648 \mathrm{~m}$ \\
\hline 1989 May 3 & 7649 & A & $5.0 \times 7.6$ & 90. & $3-5$ & 9 & $4500-5640$ & n57649a \\
\hline 1989 May 3 & 7649 & $\mathbf{P}$ & $8.0 \times 9.0$ & 90. & $2-3$ & 20 & $4400-7100$ & $\mathrm{n} 57649 \mathrm{p}$ \\
\hline 1989 May 4 & 7650 & E & $5.0 \times 11.0$ & 90. & 2 & 2 & $4810-5130$ & $\mathrm{n} 57650 \mathrm{e}$ \\
\hline 1989 May 7 & 76 & A & $5.0 \times 7.6$ & 9 & $2-3$ & 9 & $4450-5550$ & $\mathrm{n} 57653 \mathrm{aa}$ \\
\hline 1989 May 7 & 7653 & A & $1.0 \times 7.6$ & 90. & $2-3$ & 4 & $4450-5550$ & n57653ab \\
\hline 1989 May 8 & 7654 & A & $5.0 \times 7.6$ & 90. & $2-3$ & 9 & $4450-5610$ & n57654aa \\
\hline 1989 May 8 & 7654 & A & $1.0 \times 7.6$ & 90. & $2-3$ & 4 & $4450-5610$ & n57654ab \\
\hline 1989 May 8 & 7654 & $\mathrm{E}$ & $5.0 \times 13.1$ & 90. & 3 & 2 & $4790-5110$ & $\mathrm{n} 57654 \mathrm{e}$ \\
\hline 1989 May 8 & 7654 & $\mathrm{~F}$ & $3.2 \times 6.4$ & 90. & $1-2$ & 5 & $4540-7060$ & n57654f \\
\hline 1989 May 9 & 7655 & $\mathrm{Q}$ & $4.0 \times 4.0$ & 90. & $1-2$ & 9 & $3520-6560$ & $\mathrm{n} 57655 \mathrm{q}$ \\
\hline 1989 May 9 & 7655 & A & $5.0 \times 7.6$ & 90. & $4-5$ & 15 & $3400-6000$ & n57655ab \\
\hline 1989 May 9 & 7655 & A & $1.0 \times 7.6$ & 90. & $4-5$ & 11 & $3400-6000$ & n57655aa \\
\hline 1989 May 9 & 7656 & $\mathrm{M}$ & $2.4 \times 10.0$ & 0. & 2 & 2 & $4730-5300$ & $\mathrm{n} 57656 \mathrm{~m}$ \\
\hline 1989 May 10 & 7656 & $\mathrm{~F}$ & $3.2 \times 6.4$ & 90. & $1-2$ & 5 & $4540-7060$ & $\mathrm{n} 57656 \mathrm{f}$ \\
\hline 1989 May 11 & 7657 & A & $5.0 \times 7.6$ & 90. & $3-4$ & 9 & $4470-5620$ & n57657aa \\
\hline 1989 May 11 & 7657 & A & $1.0 \times 7.6$ & 90. & $3-4$ & 4 & $4470-5620$ & $\mathrm{n} 57657 \mathrm{ab}$ \\
\hline 1989 May 12 & 7658 & $\mathrm{H}$ & $2.1 \times 8.6$ & 90. & 1.5 & 7 & $4210-5810$ & n57658ha \\
\hline 1989 May 12 & 7658 & $\mathrm{H}$ & $7.0 \times 11.9$ & 90. & 1.5 & 18 & $4210-5810$ & $\mathrm{n} 57658 \mathrm{hb}$ \\
\hline 1989 May 14 & 7660 & I & $4.5 \times 27.2$ & 130. & $1.5-3$ & 14 & $3200-6070$ & n57660ia \\
\hline 1989 May 14 & 7660 & I & $4.5 \times 27.2$ & 130. & $1.5-3$ & 14 & $5740-7500$ & n57660ib \\
\hline 1989 May 14 & 7660 & I & $4.5 \times 27.2$ & 130. & $1.5-3$ & 4 & $4350-5120$ & $\mathrm{n} 57660 \mathrm{ic}$ \\
\hline 1989 May 15 & 7661 & B & $20.0 \times 28.0$ & 0. & & 10 & $4600-6980$ & $\mathrm{n} 57661 \mathrm{~b}$ \\
\hline 1989 May 16 & 7663 & M & $2.0 \times 10.0$ & 0. & 2 & 11 & $3760-7080$ & n57663ma \\
\hline 1989 May 16 & 7663 & $M$ & $3.0 \times 10.0$ & 0. & 2 & 15 & $3760-7080$ & $\mathrm{n} 57663 \mathrm{mb}$ \\
\hline
\end{tabular}


TABLE 1-Continued

\begin{tabular}{|c|c|c|c|c|c|c|c|c|}
\hline $\begin{array}{l}\text { UT } \\
\text { Date } \\
(1)\end{array}$ & $\begin{array}{c}\text { Julian Date } \\
(2440000+) \\
(2)\end{array}$ & $\begin{array}{c}\text { Code } \\
(3) \\
\end{array}$ & $\begin{array}{l}\text { Apertur } \\
\text { Size } \\
(4)\end{array}$ & $\begin{array}{l}\text { e } \\
\text { P.A. } \\
(5)\end{array}$ & $\begin{array}{l}\text { Seeing } \\
(") \\
(6) \\
\end{array}$ & $\begin{array}{c}\text { Res. } \\
(\AA) \\
(7)\end{array}$ & $\begin{array}{c}\text { Range } \\
(\AA) \\
(8) \\
\end{array}$ & $\begin{array}{l}\text { IRAF } \\
\text { file } \\
(9) \\
\end{array}$ \\
\hline 1989 May 16 & 7663 & M & $4.0 \times 10.0$ & 0. & 2 & 21 & $3760-7080$ & $\mathrm{n} 57663 \mathrm{mc}$ \\
\hline 1989 May 16 & 7663 & M & $2.0 \times 10.0$ & 90. & 2 & 11 & $3760-7080$ & n57663md \\
\hline 1989 May 16 & 7663 & M & $3.0 \times 10.0$ & 90. & 2 & 15 & $3760-7080$ & n57663me \\
\hline 1989 May 19 & 7665 & G & 7.0 round & - & $2-3$ & 8 & $4000-6540$ & n57665g \\
\hline 1989 May 21 & 7668 & $M$ & $2.1 \times 10.0$ & 0 & $3-4$ & 2 & $4750-5310$ & $\mathrm{n} 57668 \mathrm{ma}$ \\
\hline 1989 May 21 & 7668 & M & $2.1 \times 10.0$ & 0 & $3-4$ & 3 & $6490-6690$ & $\mathrm{n} 57668 \mathrm{mb}$ \\
\hline 1989 May 27 & 7673 & $\mathrm{~F}$ & $3.2 \times 6.4$ & 90. & 1 & 5 & $4140-7050$ & n57673f \\
\hline 1989 May 28 & 7674 & $\mathrm{~F}$ & $3.2 \times 6.4$ & 90. & 1 & 5 & $4140-7050$ & n57674f \\
\hline 1989 Мау 29 & 7675 & D & $1.0 \times 7.0$ & 61. & $1-1.3$ & 2 & $4850-5280$ & n57675da \\
\hline 1989 May 29 & 7675 & D & $1.0 \times 7.0$ & 61. & $1-1.3$ & 2 & $6460-7100$ & $\mathrm{n} 57675 \mathrm{db}$ \\
\hline 1989 May 30 & 7676 & D & $1.0 \times 7.0$ & 61. & $1-1.3$ & 2 & $4850-5280$ & n57676da \\
\hline 1989 May 30 & 7676 & D & $1.0 \times 7.0$ & 61. & $1-1.3$ & 2 & $6450-7100$ & $\mathrm{n} 57676 \mathrm{db}$ \\
\hline 1989 May 31 & 7678 & $\mathrm{~L}$ & 2.0 round & - & 5 & 3 & $4100-5080$ & n576781 \\
\hline 1989 Jun 1 & 7678 & A & $5.0 \times 7.6$ & 90. & $2-3$ & 9 & $4450-5400$ & n57678a \\
\hline 1989 Jun 1 & 7678 & $\mathrm{~F}$ & $3.2 \times 6.4$ & 90. & 1 & 5 & $4510-7060$ & n57678f \\
\hline 1989 Jun 1 & 7679 & $\mathrm{~L}$ & 2.0 round & - & 4 & 3 & $4110-5080$ & n57679l \\
\hline 1989 Jun 2 & 7679 & $\mathrm{~F}$ & $3.2 \times 6.4$ & 90. & 1 & 5 & $4510-7060$ & n57679f \\
\hline 1989 Jun 2 & 7680 & $\mathrm{~L}$ & 2.0 round & - & 3 & 3 & $4180-5070$ & n57680l \\
\hline 1989 Jun 3 & 7680 & $\mathrm{~F}$ & $3.2 \times 6.4$ & 90. & 1.5 & 5 & $4510-7060$ & n57680f \\
\hline 1989 Jun 3 & 7680 & $\mathrm{E}$ & $2.0 \times 12.0$ & 90. & 2 & 2 & $4790-5110$ & $\mathrm{n} 57680 \mathrm{e}$ \\
\hline 1989 Jun 4 & 7681 & $\mathrm{~F}$ & $3.2 \times 6.4$ & 90. & 1.5 & 5 & $4540-7060$ & n57681f \\
\hline 1989 Jun 4 & 7681 & $\mathrm{E}$ & $5.0 \times 12.7$ & 90. & 4 & 2 & $4830-5150$ & n57681e \\
\hline 1989 Jun 5 & 7682 & $\mathbf{F}$ & $3.2 \times 6.4$ & 90. & 1.5 & 5 & $4550-7050$ & n57682f \\
\hline 1989 Jun 5 & 7682 & $\mathrm{E}$ & $5.0 \times 18.6$ & 90. & 3 & 2 & $4830-5150$ & n57682e \\
\hline 1989 Jun 6 & 7683 & $\mathrm{~F}$ & $3.2 \times 6.4$ & 90. & 1.5 & 5 & $4550-7050$ & n57683f \\
\hline 1989 Jun 7 & 7684 & $\mathrm{~F}$ & $3.2 \times 6.4$ & 90. & 1.5 & 5 & $4550-7050$ & n57684f \\
\hline 1989 Jun 8 & 7685 & $\mathrm{~F}$ & $3.2 \times 6.4$ & 90. & 1.5 & 5 & $4550-7050$ & n57685f \\
\hline 1989 Jun 8 & 7686 & $\mathbf{M}$ & $2.1 \times 10.0$ & 90 & 2 & 2 & $4250-4800$ & n57686ma \\
\hline 1989 Jun 8 & 7686 & M & $2.1 \times 10.0$ & 90 & 2 & 3 & $6310-6810$ & $\mathrm{n} 57686 \mathrm{mb}$ \\
\hline 1989 Jun 9 & 7686 & $\mathbf{F}$ & $3.2 \times 6.4$ & 90. & 1.5 & 5 & $4550-7050$ & n57686f \\
\hline 1989 Jun 9 & 7687 & C & $1.5 \times 6.0$ & 0. & 1.2 & 4 & $3330-7320$ & n57687c \\
\hline 1989 Jun 22 & 7699 & $\mathrm{~N}$ & $8.8 \times 12.0$ & 0. & 2 & 15 & $4300-6870$ & n57699n \\
\hline 1989 Jun 23 & 7700 & $\mathrm{~N}$ & $8.8 \times 12.0$ & 0. & 3 & 10 & $4320-5970$ & n57700n \\
\hline 1989 Jun 24 & 7701 & $\mathrm{~N}$ & $8.8 \times 9.6$ & 0. & 3 & 10 & $4320-5970$ & n57701n \\
\hline 1989 Jun 25 & 7702 & $\mathbf{K}$ & $1.7 \times 3.0$ & 0. & 1.5 & 10 & $4360-7040$ & $\mathrm{n} 57702 \mathrm{k}$ \\
\hline 1989 Jun 26 & 7703 & I & $4.5 \times 27.2$ & 90. & $2-3$ & 14 & $3200-5460$ & n57703ia \\
\hline 1989 Jun 26 & 7703 & I & $4.5 \times 27.2$ & 90. & $2-3$ & 14 & $5700-8240$ & n57703ib \\
\hline 1989 Jun 26 & 7703 & $\mathbf{K}$ & $1.7 \times 3.0$ & 0. & 1.5 & 10 & $4570-7500$ & n57703k \\
\hline 1989 Jun 27 & 7704 & I & $4.5 \times 27.2$ & 90. & 2 & 4 & $4350-5060$ & n57704i \\
\hline 1989 Jun 28 & 7705 & $\mathbf{F}$ & $3.2 \times 6.4$ & 90. & 1 & 5 & $4550-7050$ & n57705f \\
\hline 1989 Jun 29 & 7706 & $\mathrm{~K}$ & $1.7 \times 3.0$ & 0. & 1.5 & 4 & $5950-7250$ & n57706k \\
\hline 1989 Jun 30 & 7707 & $\mathbf{F}$ & $3.2 \times 6.4$ & 90. & 1 & 5 & $4550-7050$ & n57707f \\
\hline $1989 \mathrm{Ju} 11$ & 7708 & $\mathbf{F}$ & $3.2 \times 6.4$ & 90. & 3 & 5 & $4550-7050$ & n57708f \\
\hline $1989 \mathrm{Jul} 1$ & 7708 & 0 & $1.9 \times 4.7$ & 90. & $1.5-2$ & 4 & $6330-7340$ & n57708o \\
\hline $1989 \mathrm{Jul} 2$ & 7709 & $\mathrm{~F}$ & $3.2 \times 6.4$ & 90. & 1 & 5 & $4550-7050$ & $\mathrm{n} 57709 \mathrm{f}$ \\
\hline $1989 \mathrm{Jul} 3$ & 7710 & $\mathbf{F}$ & $3.2 \times 6.4$ & 90. & 1 & 5 & $4550-7050$ & n57710f \\
\hline $1989 \mathrm{Jul} 3$ & 7710 & 0 & $1.9 \times 4.7$ & 90. & $1.5-2$ & 3 & $4550-5550$ & n57710o \\
\hline 1989 Jul 3 & 7711 & $\mathrm{~L}$ & 2.0 round & - & 4 & 3 & $4110-5060$ & n577111 \\
\hline 1989 Jul 4 & 7711 & $\mathrm{~A}$ & $5.0 \times 7.6$ & 90. & $2-3$ & 15 & $3400-5900$ & n57711aa \\
\hline $1989 \mathrm{Jul} 4$ & 7711 & A & $1.0 \times 7.6$ & 90. & $2-3$ & 11 & $3400-5900$ & $\mathrm{n} 57711 \mathrm{ab}$ \\
\hline 1989 Jul 5 & 7713 & M & $2.1 \times 10.0$ & 0. & 3 & 2 & $4660-5230$ & $\mathrm{n} 57713 \mathrm{~m}$ \\
\hline $1989 \mathrm{Jul} 8$ & 7715 & $\mathbf{F}$ & $3.2 \times 6.4$ & 90. & 1 & 5 & $4550-7050$ & $\mathrm{n} 57715 \mathrm{f}$ \\
\hline 1989 Jul 9 & 7716 & $\mathrm{H}$ & $4.0 \times 10.0$ & 61. & 4 & 20 & $3050-9150$ & n57716h \\
\hline 1989 Jul 12 & 7719 & A & $5.0 \times 7.6$ & 90. & $3-4$ & 15 & $4200-6820$ & n57719aa \\
\hline 1989 Jul 12 & 7719 & A & $1.0 \times 7.6$ & 90. & $3-4$ & 11 & $4200-6820$ & n57719ab \\
\hline 1989 Jul 18 & 7725 & A & $5.0 \times 7.6$ & 90. & $4-5$ & 15 & $3550-6180$ & n57725aa \\
\hline 1989 Jul 18 & 7725 & A & $1.0 \times 7.6$ & 90. & $4-5$ & 11 & $3550-6180$ & n57725ab \\
\hline $1989 \mathrm{Jul} 21$ & 7728 & $\mathrm{~N}$ & $8.8 \times 12.0$ & 0. & $2-3$ & 15 & $4350-7060$ & n57728n \\
\hline 1989 Jul 23 & 7730 & $\mathrm{~N}$ & $8.8 \times 16.8$ & 0. & $2-3$ & 15 & $4420-7050$ & $\mathrm{n} 57730 \mathrm{n}$ \\
\hline 1989 Jul 29 & 7736 & $\mathrm{H}$ & $3.0 \times 6.6$ & 62. & 1.5 & 3 & $4570-5380$ & n $57736 \mathrm{ha}$ \\
\hline 1989 Jul 29 & 7736 & $\mathrm{H}$ & $3.0 \times 6.6$ & 62. & 1.5 & 3 & $3770-4580$ & n $57736 \mathrm{hb}$ \\
\hline 1989 Aug 2 & 7741 & $\mathrm{~L}$ & 2.0 round & - & 3 & 3 & $4150-5070$ & n577411 \\
\hline 1989 Aug 3 & 7742 & $\mathrm{C}$ & $1.5 \times 6.0$ & 72.1 & 1 & 4 & $3100-7070$ & n $57742 \mathrm{c}$ \\
\hline 1989 Aug 9 & 7748 & C & $1.5 \times 6.0$ & 75.4 & $\leq 0.8$ & 4 & $3200-7380$ & n $57748 \mathrm{c}$ \\
\hline 1989 Aug 10 & 7749 & M & $1.5 \times 10.0$ & 0. & 1.5 & 4 & $4480-5600$ & $\mathrm{n} 57749 \mathrm{~m}$ \\
\hline 1989 Aug 15 & 7754 & $\mathbf{M}$ & $2.1 \times 10.0$ & 0. & 1.5 & 2 & $4710-5280$ & $\mathrm{n} 57754 \mathrm{~m}$ \\
\hline 1989 Aug 18 & 7757 & M & $2.0 \times 10.0$ & 0. & 2 & 11 & $3870-7210$ & $\mathrm{n} 57757 \mathrm{~m}$ \\
\hline 1989 Aug 19 & 7758 & M & $2.0 \times 10.0$ & 0. & 2 & 11 & $3870-7210$ & $\mathrm{n} 57758 \mathrm{~m}$ \\
\hline 1989 Aug 20 & 7759 & M & $1.0 \times 10.0$ & 0. & 1 & 2 & $4410-5290$ & $\mathrm{n} 57759 \mathrm{~m}$ \\
\hline
\end{tabular}


TABLE 1-Continued

\begin{tabular}{|c|c|c|c|c|c|c|c|c|}
\hline \multirow{2}{*}{$\begin{array}{c}\text { UT } \\
\text { Date } \\
(1) \\
\end{array}$} & \multirow{2}{*}{$\begin{array}{c}\text { Julian Date } \\
(2440000+) \\
(2)\end{array}$} & \multirow[b]{2}{*}{$\begin{array}{c}\text { Code } \\
(3)\end{array}$} & \multicolumn{2}{|c|}{ Aperture } & \multirow{2}{*}{$\begin{array}{c}\text { Seeing } \\
(") \\
(6) \\
\end{array}$} & \multirow{2}{*}{$\begin{array}{c}\text { Res. } \\
(\AA) \\
(7) \\
\end{array}$} & \multirow{2}{*}{$\begin{array}{c}\text { Range } \\
(\AA) \\
(8) \\
\end{array}$} & \multirow{2}{*}{$\begin{array}{l}\text { IRAF } \\
\text { file } \\
(9) \\
\end{array}$} \\
\hline & & & $\begin{array}{c}\text { Size } \\
(4) \\
\end{array}$ & $\begin{array}{c}\text { P.A. } \\
(5)\end{array}$ & & & & \\
\hline 1989 Aug 26 & 7765 & M & $1.0 \times 10.0$ & 0. & 1 & 2 & $4400-5270$ & $\mathrm{n} 57765 \mathrm{~m}$ \\
\hline 1989 Aug 28 & 7766 & I & $4.5 \times 27.2$ & 90. & 3 & 14 & $3200-6040$ & n57766ia \\
\hline 1989 Aug 28 & 7766 & I & $4.5 \times 27.2$ & 90. & 3 & 14 & $5700-8400$ & n57766ib \\
\hline 1989 Aug 28 & 7766 & I & $4.5 \times 27.2$ & 90. & 3 & 4 & $4350-5130$ & n57766ic \\
\hline 1989 Aug 28 & 7766 & $\mathrm{~N}$ & $8.8 \times 16.0$ & 0. & $2-3$ & 10 & $4300-6000$ & $\mathrm{n} 57766 \mathrm{n}$ \\
\hline 1989 Aug 28 & 7767 & M & $2.0 \times 10.0$ & 0. & $2-3$ & 2 & $4390-5270$ & $\mathrm{n} 57767 \mathrm{~m}$ \\
\hline 1989 Aug 29 & 7767 & I & $4.5 \times 27.2$ & 90. & 3 & 14 & $3200-6060$ & n57767ia \\
\hline 1989 Aug 29 & 7767 & I & $4.5 \times 27.2$ & 90. & 3 & 14 & $5700-8400$ & n57767ib \\
\hline 1989 Aug 29 & 7767 & I & $4.5 \times 27.2$ & 90. & 3 & 4 & $4350-5120$ & n57767ic \\
\hline 1989 Sep 8 & 7777 & $\mathrm{H}$ & $4.0 \times 10.0$ & 61. & 2 & 10 & $3400-6310$ & $\mathrm{n} 57777 \mathrm{~h}$ \\
\hline 1989 Sep 9 & 7778 & I & $4.5 \times 27.2$ & 90. & 3 & 14 & $3200-6000$ & n57778ia \\
\hline 1989 Sep 9 & 7778 & I & $4.5 \times 27.2$ & 90. & 3 & 14 & $5720-8420$ & n57778ib \\
\hline 1989 Sep 9 & 7778 & I & $4.5 \times 27.2$ & 90. & 3 & 4 & $4350-5130$ & n57778ic \\
\hline 1989 Sep 9 & 7778 & A & $5.0 \times 7.6$ & 90. & $2-3$ & 11 & $3400-5890$ & n57778a \\
\hline 1989 Sep 10 & 7779 & I & $4.5 \times 27.2$ & 90. & $1.5-2$ & 14 & $3200-6050$ & n57779ia \\
\hline 1989 Sep 10 & 7779 & I & $4.5 \times 27.2$ & 90. & $1.5-2$ & 14 & $5700-8420$ & n57779ib \\
\hline 1989 Sep 28 & 7797 & $\mathrm{H}$ & $4.0 \times 10.0$ & 59. & $3-4$ & 10 & $3400-6310$ & $\mathrm{n} 57797 \mathrm{~h}$ \\
\hline 1989 Oct 10 & 7809 & $\mathrm{H}$ & $4.0 \times 10.0$ & 60. & $1-2$ & 10 & $4700-6310$ & $\mathrm{n} 57809 \mathrm{~h}$ \\
\hline
\end{tabular}

NoTE.-Codes for Data origin (col. [3]) are as follows:

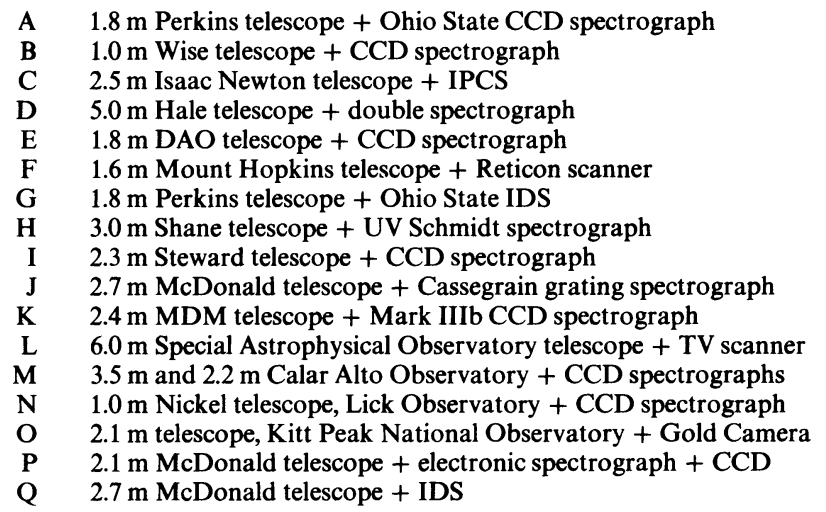

TABLE 2

Photographic Photometry

\begin{tabular}{|c|c|c|c|c|}
\hline $\begin{array}{c}\text { UT } \\
\text { Date } \\
(1)\end{array}$ & $\begin{array}{c}\text { Julian Date } \\
(2,440,000+) \\
(2)\end{array}$ & $\begin{array}{c}U \\
(3)\end{array}$ & $\begin{array}{c}B \\
(4)\end{array}$ & $\begin{array}{c}\boldsymbol{V} \\
(5)\end{array}$ \\
\hline 1988 Dec 14 & 7509 & $13.51 \pm 0.03$ & $14.11 \pm 0.09$ & $13.84 \pm 0.11$ \\
\hline 1988 Dec $18 \ldots \ldots \ldots \ldots$ & 7513 & $13.17 \pm 0.04$ & $14.14 \pm 0.14$ & $13.71 \pm 0.07$ \\
\hline 1989 Jan 27 & 7553 & $\ldots$ & $13.93 \pm 0.08$ & $\ldots$ \\
\hline 1989 Mar 11 & 7596 & $13.46 \pm 0.07$ & $14.10 \pm 0.12$ & $13.78 \pm 0.09$ \\
\hline 1989 Mar $12 \ldots \ldots \ldots$ & 7597 & $13.55 \pm 0.11$ & $13.94 \pm 0.13$ & $13.82 \pm 0.27$ \\
\hline 1989 Mar $29 \ldots \ldots$ & 7614 & $13.23 \pm 0.14$ & $13.87 \pm 0.08$ & $13.75 \pm 0.17$ \\
\hline 1989 Apr 1 & 7617 & $\ldots$ & $13.89 \pm 0.09$ & $\ldots$ \\
\hline 1989 Apr 17 & 7633 & $12.76 \pm 0.28$ & $13.68 \pm 0.10$ & $13.48 \pm 0.10$ \\
\hline 1989 Apr $25 \ldots$ & 7641 & $\ldots$ & $13.79 \pm 0.07$ & $\ldots$ \\
\hline 1989 Apr $29 \ldots \ldots \ldots$ & 7645 & $\ldots$ & $13.95 \pm 0.05$ & $\ldots$ \\
\hline 1989 May 3 & 7649 & $12.96 \pm 0.05$ & $13.90 \pm 0.09$ & $13.63 \pm 0.15$ \\
\hline 1989 May 7 & 7653 & $13.03 \pm 0.07$ & $13.98 \pm 0.06$ & $13.71 \pm 0.15$ \\
\hline 1989 May $13 \ldots$ & 7659 & $\ldots$ & $13.91 \pm 0.08$ & $\ldots$ \\
\hline 1989 May $16 \ldots \ldots$ & 7662 & $\ldots$ & $13.76 \pm 0.13$ & $\ldots$ \\
\hline 1989 May $23 \ldots \ldots \ldots$ & 7669 & $13.49 \pm 0.05$ & $13.93 \pm 021$ & $13.42 \pm 0.06$ \\
\hline 1989 May $27 \ldots$ & 7673 & $\ldots$ & $13.84 \pm 0.18$ & $\ldots$ \\
\hline $1989 \mathrm{Jul} 26 \ldots \ldots \ldots \ldots$ & 7733 & $\ldots$ & $14.56 \pm 0.24$ & $13.75 \pm 0.27$ \\
\hline
\end{tabular}


TABLE 3

Photoelectric Photometry

\begin{tabular}{|c|c|c|c|c|c|c|}
\hline $\begin{array}{l}\text { UT } \\
\text { Date } \\
(1)\end{array}$ & $\begin{array}{c}\text { Julian Date } \\
(2,440,000+) \\
(2)\end{array}$ & $\begin{array}{c}\text { Aperture } \\
\text { (3) }\end{array}$ & $\begin{array}{c}U \\
(4)\end{array}$ & $\begin{array}{c}B \\
(5)\end{array}$ & $\begin{array}{l}V \\
(6)\end{array}$ & $\begin{array}{c}R \\
(7)\end{array}$ \\
\hline 1989 Apr 6 & 7622 & $20^{\prime \prime}$ & .. & $13.64 \pm 0.01$ & $13.18 \pm 0.01$ & $13.17 \pm 0.01$ \\
\hline 1989 Apr 7 & 7623 & 16 & $\ldots$ & $13.78 \pm 0.04$ & $13.33 \pm 0.02$ & $13.30 \pm 0.02$ \\
\hline 1989 Apr 8 & 7624 & 16 & $\ldots$ & $13.73 \pm 0.07$ & $13.29 \pm 0.01$ & $13.27 \pm 0.01$ \\
\hline 1989 Apr $9 \ldots$. & 7625 & 16 & $\ldots$ & $13.75 \pm 0.02$ & $13.30 \pm 0.01$ & $13.28 \pm 0.01$ \\
\hline 1989 Apr $10 \ldots \ldots \ldots$. & 7626 & 16 & $\ldots$ & $13.76 \pm 0.03$ & $13.32 \pm 0.02$ & $13.30 \pm 0.02$ \\
\hline 1989 Jun 10 .......... & 7687 & 16 & $13.25 \pm 0.05$ & $14.13 \pm 0.04$ & $13.50 \pm 0.02$ & $13.47 \pm 0.04$ \\
\hline 1989 Jun 11 .......... & 7688 & 16 & $13.30 \pm 0.08$ & $14.18 \pm 0.03$ & $13.55 \pm 0.02$ & $13.50 \pm 0.04$ \\
\hline 1989 Jul $1 \ldots \ldots \ldots . .$. & 7708 & 16 & $13.18 \pm 0.04$ & $14.11 \pm 0.03$ & $13.48 \pm 0.02$ & $13.42 \pm 0.03$ \\
\hline
\end{tabular}

region, since these are by far the most numerous data, as well as the easiest to calibrate.

\section{a) Absolute Calibration of the Spectra}

Absolute flux calibration of optical spectra of variable AGNs can be accomplished reliably by noting that the flux in the narrow emission lines is constant over the time scales of interest in this study. The large spatial extent of the narrowline region (NLR) and the low electron density (which implies a very long recombination time) tend to damp out the effect of any short-term variability of the ionizing continuum. We thus use the strong, narrow [O III] $\lambda 5007$ as an internal flux standard for variability studies. We note that the relative spectral energy distribution in the observed spectrum is calibrated by referencing the data to observations of a known standard star, usually Feige 98 (Stone 1977) in the case of these observations, but the absolute calibration of the spectrum is given by multiplying the data by a constant factor to give the correct [O III] $\lambda 5007$ flux.

TABLE 4

\section{CCD PHOTOMETRY}

\begin{tabular}{|c|c|c|}
\hline $\begin{array}{l}\text { UT } \\
\text { Date } \\
(1)\end{array}$ & $\begin{array}{c}\text { Julian Date } \\
(2,440,000+) \\
(2)\end{array}$ & $\begin{array}{c}B \\
(3)\end{array}$ \\
\hline 1989 Mar 8 & 7593 & $14.154 \pm 0.044$ \\
\hline 1989 Mar 27 & 7612 & $13.901 \pm 0.016$ \\
\hline 1989 Apr 9. & 7625 & $13.810 \pm 0.012$ \\
\hline 1989 Apr 17 & 7633 & $13.853 \pm 0.015$ \\
\hline 1989 Apr 22 & 7638 & $13.834 \pm 0.021$ \\
\hline 1989 Apr 24 & 7640 & $13.792 \pm 0.007$ \\
\hline 1989 May 18 & 7664 & $13.886 \pm 0.020$ \\
\hline 1989 May 26 & 7672 & $13.885 \pm 0.072$ \\
\hline 1989 May 27 & 7673 & $13.943 \pm 0.006$ \\
\hline 1989 May 29 & 7675 & $13.952 \pm 0.007$ \\
\hline 1989 Jun $3 \ldots$ & 7680 & $13.950 \pm 0.012$ \\
\hline 1989 Jun 12 & 7689 & $14.072 \pm 0.014$ \\
\hline 1989 Jun 18 & 7695 & $14.021 \pm 0.032$ \\
\hline 1989 Jun 29 & 7706 & $14.048 \pm 0.022$ \\
\hline $1989 \mathrm{Jul} 3 \ldots$ & 7710 & $14.007 \pm 0.018$ \\
\hline 1989 Jul 7 & 7714 & $13.980 \pm 0.009$ \\
\hline 1989 Jul 9 & 7716 & $13.978 \pm 0.015$ \\
\hline 1989 Jul $12 \ldots$ & 7719 & $13.966 \pm 0.013$ \\
\hline 1989 Jul $19 \ldots$ & 7726 & $14.040 \pm 0.040$ \\
\hline 1989 Jul 24 ........... & 7731 & $14.110 \pm 0.030$ \\
\hline $1989 \mathrm{Jul} 31 \ldots \ldots \ldots \ldots$ & 7738 & $14.270 \pm 0.020$ \\
\hline 1989 Aug 6 .. & 7744 & $14.270 \pm 0.020$ \\
\hline 1989 Aug 7. & 7745 & $14.220 \pm 0.024$ \\
\hline 1989 Aug 9 & 7747 & $14.170 \pm 0.040$ \\
\hline 1989 Aug 25 & 7763 & $14.164 \pm 0.010$ \\
\hline $1989 \operatorname{Sep} 2 \ldots$ & 7771 & $14.101 \pm 0.019$ \\
\hline $1989 \operatorname{Sep} 3$ & 7772 & $14.024 \pm 0.017$ \\
\hline
\end{tabular}

The absolute flux in the [O III] $\lambda 5007$ line is obtained by averaging measurements made through large spectrograph entrance apertures on photometric nights. The data from Table 1 which meet these criteria are listed in Table 5.

It is worth noting that one set of data, that from Wise Observatory, was treated in a slightly different fashion. These data were obtained with a very long slit instrument which was rotated to accept the light from both the target galaxy and star 1 of Penston, Penston, and Sandage (1971). This can be an especially accurate means of flux calibration (Maoz et al. 1990). The data can then be placed on an absolute flux scale once the flux of the nearby star is accurately measured. All [O III] $\lambda 5007$ flux measurements from the Wise Observatory spectra are thus averaged together in Table 5, since they constitute a single determination which, as can be seen, is in excellent agreement with the mean of the other measurements.

\section{b) Spectral Measurements}

As we will discuss below, each set of data was treated separately to minimize aperture effects. Each set of data, as designated by the individual codes in column (3) of Table 1, was treated as being homogeneous. By making the well-justified

TABLE 5

Absolute Calibration of [O III] $\lambda 5007$

\begin{tabular}{|c|c|c|}
\hline $\begin{array}{c}F([\mathrm{O} \mathrm{III}] \lambda 5007) \\
\left(10^{-13} \mathrm{ergs}_{(1)}^{\left.\operatorname{erg} \mathrm{cm}^{-1}\right)}\right.\end{array}$ & $\begin{array}{c}\text { Aperture Size } \\
\text { (2) }\end{array}$ & $\begin{array}{c}\text { Source/File Name } \\
\text { (3) }\end{array}$ \\
\hline 6.06 & $4 " .5 \times 27^{\prime \prime} .2$ & n57524ia \\
\hline $5.51 \ldots$ & $4.5 \times 27.2$ & n57524ic \\
\hline $6.01 \ldots$ & $4.5 \times 27.2$ & n57525ia \\
\hline $5.71 \ldots$ & $4.0 \times 10.0$ & n57530ha \\
\hline $5.60 \ldots$ & $15.0 \times 10.0$ & $\mathrm{n} 57530 \mathrm{hb}$ \\
\hline 5.45 & $7.0 \times 7.9$ & $\mathrm{n} 57556 \mathrm{hb}$ \\
\hline 5.36 & $4.5 \times 27.2$ & n57574ia \\
\hline 5.49 & $4.5 \times 27.2$ & n57574ib \\
\hline 5.19 & $7.0 \times 7.2$ & $\mathrm{n} 57592 \mathrm{j}$ \\
\hline $5.85 \ldots$ & $5.0 \times 7.6$ & n57606a \\
\hline $6.11 \ldots$ & $4.0 \times 10.0$ & $\mathrm{n} 57614 \mathrm{~h}$ \\
\hline $5.22 \ldots$ & $4.0 \times 10.0$ & $\mathrm{n} 57643 \mathrm{~h}$ \\
\hline $5.28 \ldots$ & $7.0 \times 11.9$ & $\mathrm{n} 7658 \mathrm{hb}$ \\
\hline $5.48 \ldots$ & $4.5 \times 27.2$ & n57703ia \\
\hline $5.77 \ldots$ & $4.0 \times 10.0$ & n57716h \\
\hline $5.52 \ldots$ & $4.5 \times 27.2$ & n57778ia \\
\hline $5.44 \ldots$ & $4.5 \times 27.2$ & n57778ic \\
\hline $5.50 \ldots$ & $4.5 \times 27.2$ & n57779ia \\
\hline $5.53 \pm 0.31^{\mathrm{a}}$ & $20.0 \times 28.0$ & $\ldots$ \\
\hline $5.58 \pm 0.27^{\mathrm{b}} \ldots \ldots \ldots \ldots \ldots \ldots$ & $\ldots$ & $\ldots$ \\
\hline
\end{tabular}

a Mean of Wise spectra (see text).

b Mean value (adopted absolute flux) 
(Table 5) assumption that the narrow-line flux $F([\mathrm{O} \mathrm{III}]$ 25007) is constant over the time scales of interest, variability in the continuum and the $\mathrm{H} \beta$ emission line can be discerned by measuring the ratios $F_{\lambda} / F([\mathrm{O} \mathrm{III}] 25007)$ and $F(\mathrm{H} \beta) / F([\mathrm{O} \mathrm{III}]$ 25007), respectively, where $F_{\lambda}$ refers to the continuum flux at some specified wavelength and $F(\mathrm{H} \beta)$ is the integrated flux in the $\mathrm{H} \beta$ line. Since we are interested only in the variability of the continuum and emission lines, the details of how the measurements are done are less important than doing them in a systematic way. We therefore opted to make the simplest possible measurement of the $\mathrm{H} \beta$ flux by interpolating a continuum underneath the broad $\mathrm{H} \beta$ feature from the local depression between $\mathrm{H} \beta$ and the $\mathrm{He}$ II $\lambda 4686+\mathrm{Fe}$ II $\lambda 4570$ blend (i.e., at about $4785 \AA$ in the rest-frame of NGC 5548) to the local minimum between [O III] $\lambda 5007$ and the Fe II $\lambda 5250$ blend (at $\sim 5100 \AA$ ). The $\mathrm{H} \beta$ flux is taken to be the total flux above this continuum between the shortward limit and just shortward of [O III] $\lambda 4959$ (i.e., $\sim 4940 \AA$ ). We then take as the continuum measurement the value of the interpolated continuum underneath $\mathrm{H} \beta$ at a point halfway between the integration limits, i.e., at about $4870 \AA$. The continuum and integration limits are shown graphically for a sample spectrum in Figure 1.

Strictly speaking, neither of the measured quantities is very accurate on an absolute scale, although these measurements have the great virtue of being unambiguous and highly repeatable. The continuum measurement is affected by the considerable starlight contribution and heavily blended emission features. The $\mathrm{H} \beta$ measurement includes the narrow-line component $[\sim 0.1 F([\mathrm{O} \mathrm{III}] 25007)]$ and contaminating emission from blends of $\mathrm{Fe}$ II. A careful deconvolution of the $\mathrm{Fe}$ II emissionindicates that removal of these latter contaminants would decrease our measurements of $F(\mathrm{H} \beta)$ systematically by about $5 \%$. We note that repeated measurements using slightly different continua and integration limits affect the measurements typically at about the $2 \%$ level. The uncertainties in the measured quantities will be discussed in more detail in the next section.

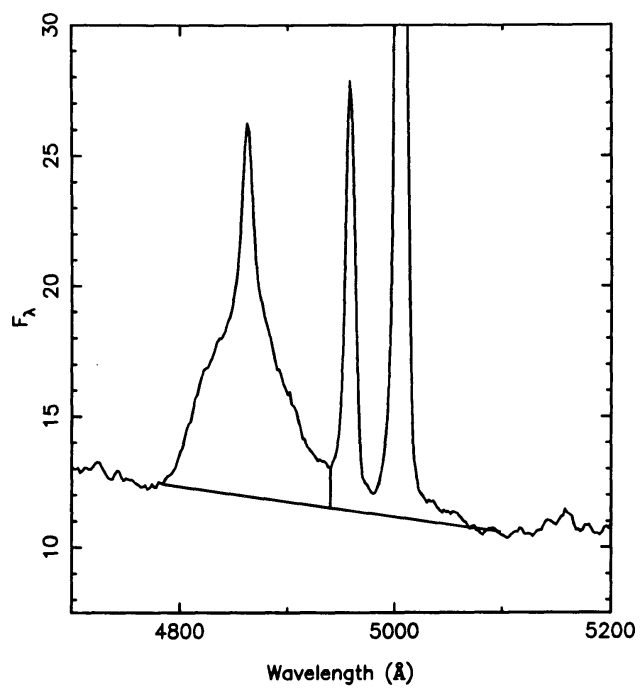

FIG. 1.-Expanded view of the spectral region near $\mathrm{H} \beta$ shown to illustrate how the spectral features are measured. A straight pseudocontinuum is drawn from the local minima just shortward and just longward of the $\mathrm{H} \beta+[\mathrm{O} \mathrm{III}]$ blend. The $\mathrm{H} \beta$ flux is taken to be the integral above this line from the shortward limit to the vertical line immediately shortward of [O III] $\lambda 4959$, and the continuum flux $F_{\lambda}(4870 \AA)$ is the pseudocontinuum value at $4870 \AA$.
The measured values of $F_{\lambda}(4870 \AA) / F([\mathrm{O}$ III $] \lambda 5007)$ and $F(\mathrm{H} \beta) / F([\mathrm{O} \mathrm{III}] 25007)$, grouped into data sets which are regarded as homogeneous, are given in Table 6. Column (5) of Table 6 points out a few cases which merit special attention. First, we note that the Wise Observatory spectra are already on a reliable flux scale. Therefore, the ratios given in columns (2) and (3) of Table 6 for the Wise Observatory data are computed using the adopted mean value for $F([\mathrm{O}$ III $] \lambda 5007)$ given in Table 5. In a few cases we were unable to use [O III] $\lambda 5007$ for flux calibration, either because it was saturated, clearly corrupted by an instrumental effect or cosmic-ray or other non-Poissonian source of noise, or in some cases because it lies beyond the long-wavelength cutoff of the spectrum. In these cases we used [O III] $\lambda 4959$ as a flux standard, and inferred a value for $F([\mathrm{O} \mathrm{III}] 25007)$ based on the mean value of the $F([\mathrm{O}$ III $] 25007) / F([\mathrm{O}$ III] 24959$)$ flux ratio measured from other spectra within the same set (note that blending and resolution effects tend to result in departures of $F([\mathrm{O} \mathrm{III}]$ $\lambda 5007) / F([\mathrm{O} \mathrm{III}] 24959)$ from its theoretical value of 2.96$)$. In cases where the longward point we use for defining the continuum lies off the spectrum, we extrapolated the continuum beyond the limit of the spectrum by using other spectra taken within one or two days as a guide. Finally, in some of the low-resolution spectra, [O III] $\lambda \lambda 4959,5007$ are very heavily blended. In these spectra, both lines were measured together and $F([\mathrm{O} \mathrm{III}] 25007)$ was taken to be $75 \%$ of the total flux in the blend.

\section{c) Intercalibration of the Data}

The basic problem with using the [O $\mathrm{III}] \lambda 5007$ flux to calibrate spectra internally is that, in contrast to the pointlike BLR and nonstellar continuum source, the NLR is sometimes partially spatially resolved. In the case of NGC 5548, Wilson et al. (1989) show that narrow-line emission is detectable as far as $\sim 15^{\prime \prime}$ from the nucleus. This means that the amount of NLR flux that is detected is a function of the size of the spectrograph entrance aperture. The problem is exacerbated by changes in seeing, since the observed surface brightness profile of the NLR is functionally different from the point-spread funotion (representing the BLR and nuclear continuum source), and the ratio of the integrals of these distributions over the aperture fluctuates most radically with seeing changes when the aperture size and the NLR size are comparable. Thus, the different aperture geometries used in these observations introduce systematic differences among the various data sets, and variations due to seeing contribute substantially to the uncertainties. Furthermore, the starlight from the host galaxy in NGC 5548 contributes substantially to the total continuum observed through the apertures used in this study, and clearly the amount of detected starlight is a strong function of spectrograph entrance aperture.

We implicitly assume that each of the individual data sets can be treated as internally homogeneous and that the differences between the data sets are attributable to aperture effects. The justification for this is twofold: (1) The larger individual data sets all show the same pattern of variability as seen in the IUE data of Paper I, and (2) if we consider the data sets which are the most similar in terms of spectrograph entrance aperture, spectral resolution, and signal-to-noise ratio, the differences between the measured values of $F_{\lambda}(4870 \AA) / F([\mathrm{O}$ III] $\lambda 5007)$ and $F(\mathrm{H} \beta) / F([\mathrm{O}$ III $] \lambda 5007)$ are very slight. To illustrate the latter point, unadjusted measurements of $F_{\lambda}(4870 \AA)$ and $F(\mathrm{H} \beta)$ from the $1.8 \mathrm{~m}$ Perkins telescope (sets $\mathrm{A}$ and $\mathrm{G}$ ), the 
TABLE 6

Measurements of Spectra

\begin{tabular}{llll|} 
& & MEASUREMENT \\
\hline
\end{tabular}

$\begin{array}{lll}F([\mathrm{O} \mathrm{III}] \lambda 5007) & F([\mathrm{O} \mathrm{III}] \lambda 5007)\end{array}$

\begin{tabular}{|c|c|c|c|c|c|c|c|c|c|}
\hline $\begin{array}{c}(2440000+) \\
(1)\end{array}$ & 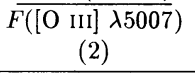 & $\begin{array}{c}F([\mathrm{O} \mathrm{III}] \lambda 5007) \\
\cdot(3)\end{array}$ & $\begin{array}{l}\text { file } \\
(4)\end{array}$ & $\begin{array}{l}\text { Notes } \\
(5)\end{array}$ & $\begin{array}{c}(2440000+) \\
(1)\end{array}$ & $\begin{array}{c}F([\mathrm{O} \mathrm{III}] \lambda 5007) \\
(2)\end{array}$ & 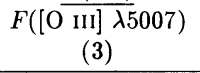 & $\begin{array}{l}\text { file } \\
(4)\end{array}$ & $\begin{array}{l}\text { Notes } \\
(5)\end{array}$ \\
\hline \multicolumn{5}{|c|}{ A - Ohio State CCD } & \multicolumn{5}{|c|}{$\mathrm{F}-\mathrm{SAO}$ Reticon (cont.) } \\
\hline 7517 & 1.98 & 1.44 & n57515a & & 7617 & 1.98 & 1.63 & n57617f & \\
\hline 7534 & 2.06 & 1.48 & n57534a & & 7618 & 2.04 & 1.63 & $\mathrm{n} 57618 \mathrm{f}$ & \\
\hline 7535 & 2.10 & 1.50 & $\mathrm{n} 57535 \mathrm{a}$ & & 7620 & 2.09 & 1.62 & $\mathrm{n} 57620 \mathrm{f}$ & \\
\hline 7560 & 1.79 & 1.63 & $\mathrm{n} 57560 \mathrm{a}$ & & 7621 & 2.10 & 1.54 & n57621f & \\
\hline 7573 & 1.70 & 1.60 & n57573a & & 7623 & 2.03 & 1.79 & n57623f & \\
\hline 7582 & 1.56 & 1.44 & n57582a & & 7624 & 2.21 & 1.61 & n57624f & \\
\hline 7589 & 1.83 & 1.42 & $\mathrm{n} 57589 \mathrm{a}$ & & 7626 & 2.08 & 1.65 & n57626f & \\
\hline 7590 & 1.87 & 1.41 & n57590a & & 7627 & 2.14 & 1.58 & $\mathrm{n} 57627 \mathrm{f}$ & \\
\hline 7600 & 2.00 & 1.23 & n57600a & & 7643 & 2.17 & 1.86 & n57643f & \\
\hline 7606 & 2.09 & 1.39 & n $57606 a$ & & 7644 & 2.20 & 1.86 & $\mathrm{n} 57644 \mathrm{f}$ & \\
\hline 7618 & 2.30 & 1.46 & $\mathrm{n} 57618 \mathrm{a}$ & & 7650 & 2.18 & 1.91 & n57650f & \\
\hline 7627 & 2.40 & 1.66 & n57627a & & 7654 & 2.02 & 1.84 & n57654f & \\
\hline 7642 & 2.50 & 1.74 & n57642a & & 7656 & 2.07 & 1.95 & $\mathrm{n} 57656 \mathrm{f}$ & \\
\hline 7649 & 2.36 & 1.77 & n57649a & & 7673 & 1.78 & 1.87 & $\mathrm{n} 57673 \mathrm{f}$ & \\
\hline 7653 & 2.36 & 1.80 & n57653aa & & 7674 & 1.76 & 1.89 & $\mathrm{n} 57674 \mathrm{f}$ & \\
\hline 7654 & 2.35 & 1.74 & n57654aa & & \multicolumn{5}{|c|}{$\mathrm{G}$ - Ohio State IDS } \\
\hline 7655 & 2.32 & 1.78 & n57655ab & & 7665 & 2.22 & 1.70 & $\mathrm{n} 57665 \mathrm{~g}$ & \\
\hline 7657 & 2.28 & 1.79 & n57657aa & & \multicolumn{5}{|c|}{ H1 - Lick Shane CCD (Large Aperture) } \\
\hline 7678 & 1.98 & 1.67 & n $57678 \mathrm{a}$ & & 7509 & 1.92 & 1.35 & n57509hc & \\
\hline 7711 & 2.07 & 1.57 & n57711aa & & 7530 & 1.97 & 1.45 & n57530ha & \\
\hline 7719 & 2.03 & 1.56 & n57719aa & & 7556 & 2.21 & 1.55 & $\mathrm{n} 57566 \mathrm{hb}$ & \\
\hline 7725 & 1.97 & 1.69 & n57725aa & & 7614 & 2.31 & 1.54 & $\mathrm{n} 57614 \mathrm{~h}$ & \\
\hline 7778 & 2.02 & 1.42 & $\mathrm{n} 57778 \mathrm{a}$ & & 7628 & 2.54 & 1.56 & $\mathrm{n} 57628 \mathrm{hb}$ & \\
\hline \multicolumn{5}{|c|}{ B - Wise Observatory CCD } & 7643 & 2.34 & 1.73 & $\mathrm{n} 57643 \mathrm{~h}$ & \\
\hline 7573 & 2.73 & 1.59 & $\mathrm{n} 57573 \mathrm{~b}$ & $\mathrm{~A}$ & 7658 & 2.50 & 1.72 & $\mathrm{n} 57658 \mathrm{hb}$ & \\
\hline 7584 & 2.73 & 1.38 & n57584b & $\mathrm{A}$ & 7716 & 2.00 & 1.53 & $\mathrm{n} 577716 \mathrm{~h}$ & \\
\hline 7644 & 3.45 & 1.70 & n $57644 b$ & A & 7777 & 1.86 & 1.37 & $\mathrm{n} 57777 \mathrm{~h}$ & \\
\hline 7661 & 3.26 & 1.70 & $\mathrm{n} 57661 \mathrm{~b}$ & A & 7797 & 2.21 & 1.53 & $\mathrm{n} 57797 \mathrm{~h}$ & \\
\hline \multicolumn{5}{|c|}{$\mathrm{C}$ - INT IPCS } & 7809 & 2.09 & 1.57 & $\mathrm{n} 57809 \mathrm{~h}$ & \\
\hline 7687 & 1.84 & 1.54 & n57687c & $\mathrm{B}$ & \multicolumn{5}{|c|}{ H2 - Lick Shane CCD (Small Aperture) } \\
\hline 7742 & 1.24 & 1.53 & n $57742 \mathrm{c}$ & & 7556 & 1.86 & 1.65 & n57556ha & \\
\hline 7748 & 1.38 & 1.59 & n57748c & & 7621 & 2.50 & 1.72 & $\mathrm{n} 57621 \mathrm{~h}$ & \\
\hline \multicolumn{5}{|c|}{ D - Palomar Double Spectrograph } & 7628 & 2.23 & 1.60 & n57628ha & \\
\hline 7616 & 1.98 & 1.67 & n57616db & $\mathrm{B}$ & 7658 & 2.02 & 1.82 & n57658ha & \\
\hline 7675 & 1.54 & 1.96 & n57675da & $\mathrm{C}$ & 7736 & 1.25 & 1.54 & n57736ha & \\
\hline 7676 & 1.62 & 1.85 & n $57676 \mathrm{da}$ & $\mathrm{C}$ & \multicolumn{5}{|c|}{ I - Steward CCD } \\
\hline \multicolumn{5}{|c|}{$\mathrm{E}-\mathrm{DAO}$ CCD } & 7524 & 2.11 & 1.64 & n57524ia & \\
\hline 7561 & 1.95 & 1.70 & $\mathrm{n} 57561 \mathrm{e}$ & & 7525 & 2.23 & 1.62 & n57525ia & \\
\hline 7564 & 1.92 & 1.64 & $\mathrm{n} 57564 \mathrm{e}$ & & 7574 & 1.89 & 1.57 & n57574ia & \\
\hline 7565 & 1.93 & 1.69 & $\mathrm{n} 57565 \mathrm{e}$ & & 7613 & 2.44 & 1.49 & n57613ia & \\
\hline 7567 & 1.89 & 1.61 & $\mathrm{n} 57567 \mathrm{e}$ & & 7645 & 2.46 & 1.84 & n57645ia & \\
\hline 7568 & 1.84 & 1.52 & $\mathrm{n} 57568 \mathrm{e}$ & & 7660 & 2.21 & 1.79 & n57660ia & \\
\hline 7572 & 1.72 & 1.59 & $\mathrm{n} 57572 \mathrm{t}$ & & 7703 & 1.96 & 1.64 & n57703ia & \\
\hline 7629 & 2.37 & 1.51 & $\mathrm{n} 57629 \mathrm{e}$ & & 7766 & 1.85 & 1.33 & n57766ia & \\
\hline 7650 & 2.36 & 1.87 & $\mathrm{n} 57650 \mathrm{e}$ & $\mathrm{C}$ & 7767 & 1.85 & 1.31 & n57767ia & \\
\hline 7654 & 2.46 & 1.85 & $\mathrm{n} 57654 \mathrm{e}$ & $\mathrm{C}$ & 7778 & 2.10 & 1.48 & n57778ia & \\
\hline 7681 & 1.94 & 1.67 & n57681e & C & 7779 & 2.10 & 1.46 & n57779ia & \\
\hline 7682 & 2.12 & 1.67 & $\mathrm{n} 57682 \mathrm{e}$ & $\mathrm{C}$ & \multicolumn{5}{|c|}{$\mathrm{J}-$ McDonald 2.7m CCDD } \\
\hline \multicolumn{5}{|c|}{$\mathrm{F}-\mathrm{SAO}$ Reticon } & 7592 & 1.96 & 1.23 & $\mathrm{n} 57592 \mathrm{j}$ & \\
\hline 7528 & 1.72 & 1.60 & $\mathrm{n} 57528 \mathrm{f}$ & & 7593 & 2.00 & 1.27 & $\mathrm{n} 57593 \mathrm{j}$ & \\
\hline 7533 & 1.78 & 1.64 & $\mathrm{n} 57533 \mathrm{f}$ & & & K1 - Michigan & CDD (Large Apert & ure) & \\
\hline 7539 & 1.88 & 1.53 & $\mathrm{n} 57539 \mathrm{f}$ & & 7600 & 1.90 & 1.30 & $\mathrm{n} 57600 \mathrm{k}$ & \\
\hline 7543 & 1.85 & 1.53 & n57543f & & & K2-Michigan & CCD (Small Apert & ure) & \\
\hline 7570 & 1.56 & 1.77 & $\mathrm{n} 57570 \mathrm{f}$ & & 7599 & 1.62 & 1.34 & $\mathrm{n} 57599 \mathrm{~kb}$ & \\
\hline 7571 & 1.59 & 1.64 & n57571f & & 7601 & 1.60 & 1.28 & $\mathrm{n} 57601 \mathrm{k}$ & \\
\hline 7587 & 1.52 & 1.56 & $\mathrm{n} 57587 \mathrm{f}$ & & 7623 & 2.55 & 1.57 & n57623k & \\
\hline 7591 & 1.53 & 1.48 & n57591: & & 7624 & 2.40 & 1.65 & $\mathrm{n} 57624 \mathrm{k}$ & \\
\hline 7592 & 1.69 & 1.54 & n57592f & & 7626 & 2.46 & 1.60 & $\mathrm{n} 57626 \mathrm{k}$ & \\
\hline 7593 & 1.51 & 1.53 & $\mathrm{n} 57593 \mathrm{fa}$ & & 7702 & 1.61 & 1.52 & $\mathrm{n} 57702 \mathrm{k}$ & \\
\hline 7593 & 1.47 & 1.50 & $\mathrm{n} 57593 \mathrm{fb}$ & & 7703 & 1.50 & 1.59 & $\mathrm{n} 57703 \mathrm{k}$ & \\
\hline 7598 & 1.67 & 1.49 & $\mathrm{n} 57598 \mathrm{f}$ & & & - Special Astroph & sical Observatory & Scanner & \\
\hline 7599 & 1.72 & 1.39 & n57599f & & 7590 & 1.73 & 1.58 & n575901 & $\mathrm{B}, \mathrm{C}$ \\
\hline 7615 & 1.90 & 1.48 & $\mathrm{n} 57615 \mathrm{f}$ & & 7678 & 1.88 & 1.68 & n576781 & $\mathrm{B}, \mathrm{C}$ \\
\hline
\end{tabular}


TABLE 6-Continued

\begin{tabular}{|c|c|c|c|c|}
\hline $\begin{array}{c}\text { Julian Date } \\
(2440000+) \\
\text { (1) }\end{array}$ & $\frac{100 F_{\lambda}(4870 \AA)}{F\left(\left[\mathrm{O}_{\mathrm{III}]} \lambda 5007\right)\right.}$ & $F\left(\left[\mathrm{O} \frac{F(\mathrm{H} \beta)}{(3) \lambda 5007)}\right.\right.$ & $\begin{array}{l}\text { IRAF } \\
\text { file } \\
(4)\end{array}$ & $\begin{array}{l}\text { Notes } \\
(5)\end{array}$ \\
\hline \multicolumn{5}{|c|}{ L - Special Astrophysical Observatory Scanner (cont.) } \\
\hline 7679 & 1.75 & 1.74 & n576791 & $\mathrm{B}, \mathrm{C}$ \\
\hline 7680 & 1.60 & 1.94 & n576801 & $\mathrm{B}, \mathrm{C}$ \\
\hline 7711 & 1.96 & 1.62 & n577111 & $\mathrm{B}, \mathrm{C}$ \\
\hline 7741 & 1.10 & 1.55 & n577411 & $\mathrm{B}$ \\
\hline \multicolumn{5}{|c|}{$\mathrm{M}$ - Calar Alto CCD } \\
\hline 7512 & 1.70 & 1.42 & n57512ma & \\
\hline 7539 & 2.25 & 1.66 & $\mathrm{n} 57539 \mathrm{~m}$ & B \\
\hline 7546 & 2.55 & 1.56 & $\mathrm{n} 57546 \mathrm{~m}$ & \\
\hline 7549 & 2.11 & 1.60 & $\mathrm{n} 57549 \mathrm{~m}$ & \\
\hline 7560 & 1.96 & 1.51 & $\mathrm{n} 57560 \mathrm{~m}$ & \\
\hline 7573 & 1.45 & 1.58 & $\mathrm{n} 57573 \mathrm{~m}$ & \\
\hline 7574 & 1.63 & 1.49 & $\mathrm{n} 57574 \mathrm{~m}$ & \\
\hline 7575 & 1.46 & 1.50 & $\mathrm{n} 57575 \mathrm{ma}$ & \\
\hline 7576 & 1.44 & 1.47 & $\mathrm{n} 57576 \mathrm{~m}$ & \\
\hline 7587 & 1.55 & 1.39 & $\mathrm{n} 57587 \mathrm{~m}$ & \\
\hline 7589 & 1.44 & 1.36 & $\mathrm{n} 57589 \mathrm{~m}$ & \\
\hline 7592 & 1.66 & 1.37 & $\mathrm{n} 57592 \mathrm{~m}$ & \\
\hline 7594 & 1.64 & 1.21 & $\mathrm{n} 57594 \mathrm{~m}$ & \\
\hline 7597 & 1.82 & 1.33 & $\mathrm{n} 57597 \mathrm{~m}$ & \\
\hline 7601 & 1.81 & 1.30 & $\mathrm{n} 57601 \mathrm{~m}$ & \\
\hline 7606 & 2.12 & 1.32 & $\mathrm{n} 57606 \mathrm{~m}$ & \\
\hline 7644 & 2.43 & 1.56 & $\mathrm{n} 57644 \mathrm{~m}$ & \\
\hline 7648 & 2.27 & 1.66 & n $57648 \mathrm{~m}$ & \\
\hline 7652 & 2.06 & 1.77 & $\mathrm{n} 57652 \mathrm{~m}$ & \\
\hline 7656 & 2.09 & 1.74 & $\mathrm{n} 57656 \mathrm{~m}$ & \\
\hline 7663 & 2.06 & 1.82 & $\mathrm{n} 57663 \mathrm{ma}$ & \\
\hline 7668 & 2.00 & 1.67 & $\mathrm{n} 57668 \mathrm{ma}$ & \\
\hline \multicolumn{5}{|c|}{ N1 - Lick Nickel CCD (Large Aperture) } \\
\hline 7699 & 2.55 & 1.55 & n57699n & \\
\hline 7700 & 2.61 & 1.55 & n57700n & \\
\hline 7701 & 2.64 & 1.57 & n57701n & \\
\hline 7728 & 2.29 & 1.60 & n57728n & \\
\hline 7730 & 2.47 & 1.55 & n57730n & \\
\hline 7766 & 2.01 & 1.44 & n57766n & \\
\hline \multicolumn{5}{|c|}{ N2 - Lick Nickel CCD (Small Aperture) } \\
\hline 7583 & 1.82 & 1.43 & n57583n & \\
\hline 7631 & 2.84 & 1.66 & $\mathrm{n} 57631 \mathrm{n}$ & \\
\hline \multicolumn{5}{|c|}{$\mathrm{O}-\mathrm{KPNO} \mathrm{CCD}$} \\
\hline 7710 & 1.63 & 1.41 & n57710o & \\
\hline \multicolumn{5}{|c|}{$\mathrm{P}-\mathrm{McDonald} 2.1 \mathrm{~m}$ CCD } \\
\hline 7631 & 2.66 & 1.51 & n57631p & $\mathrm{D}$ \\
\hline 7632 & 2.50 & 1.48 & n57632p & $\mathrm{D}$ \\
\hline 7649 & 2.43 & 1.49 & $\mathrm{n} 57649 \mathrm{p}$ & $\mathrm{D}$ \\
\hline \multicolumn{5}{|c|}{$\mathrm{Q}-$ McDonald $2.7 \mathrm{~m}$ IDS } \\
\hline 7620 & 2.10 & 1.43 & $\mathrm{n} 57620 \mathrm{q}$ & \\
\hline 7622 & 2.06 & 1.49 & n57622q & \\
\hline 7655 & 2.00 & 1.80 & $\mathrm{n} 57655 \mathrm{q}$ & \\
\hline
\end{tabular}

NOTES.-(A) Spectrum calibrated in absolute units by relative spectrophotometry (see text). Flux ratios computed using $F([\mathrm{O}$ III $] \lambda 5007)$ from Table 5. (B) $[\mathrm{O} \mathrm{III}] \lambda 5007$ unusable; calibration based on [O III] $\lambda 4959$. (C) Redward continuum point off of spectrum; continuum extrapolated. (D) [O III] $\lambda \lambda 4959,5007$ strongly blended; [O III] $\lambda 5007$ taken to be 0.75 of total blend flux.

large-aperture data from the $3 \mathrm{~m}$ Lick Shane telescope (set H1), the $2.3 \mathrm{~m}$ Steward telescope data (set I), the $2.7 \mathrm{~m} \mathrm{McDonald}$ telescope CCD data (set J), and the single large-aperture observation from the $2.4 \mathrm{~m}$ MDM telescope (set K1) are shown in Figure 2.

We can make an empirical correction for these aperture effects by comparing data from different sets which are nearly contemporaneous. If we compare closely spaced measurements of $F(\mathrm{H} \beta) / F([\mathrm{O} \mathrm{III}] 25007)$ from different data sets, and assume

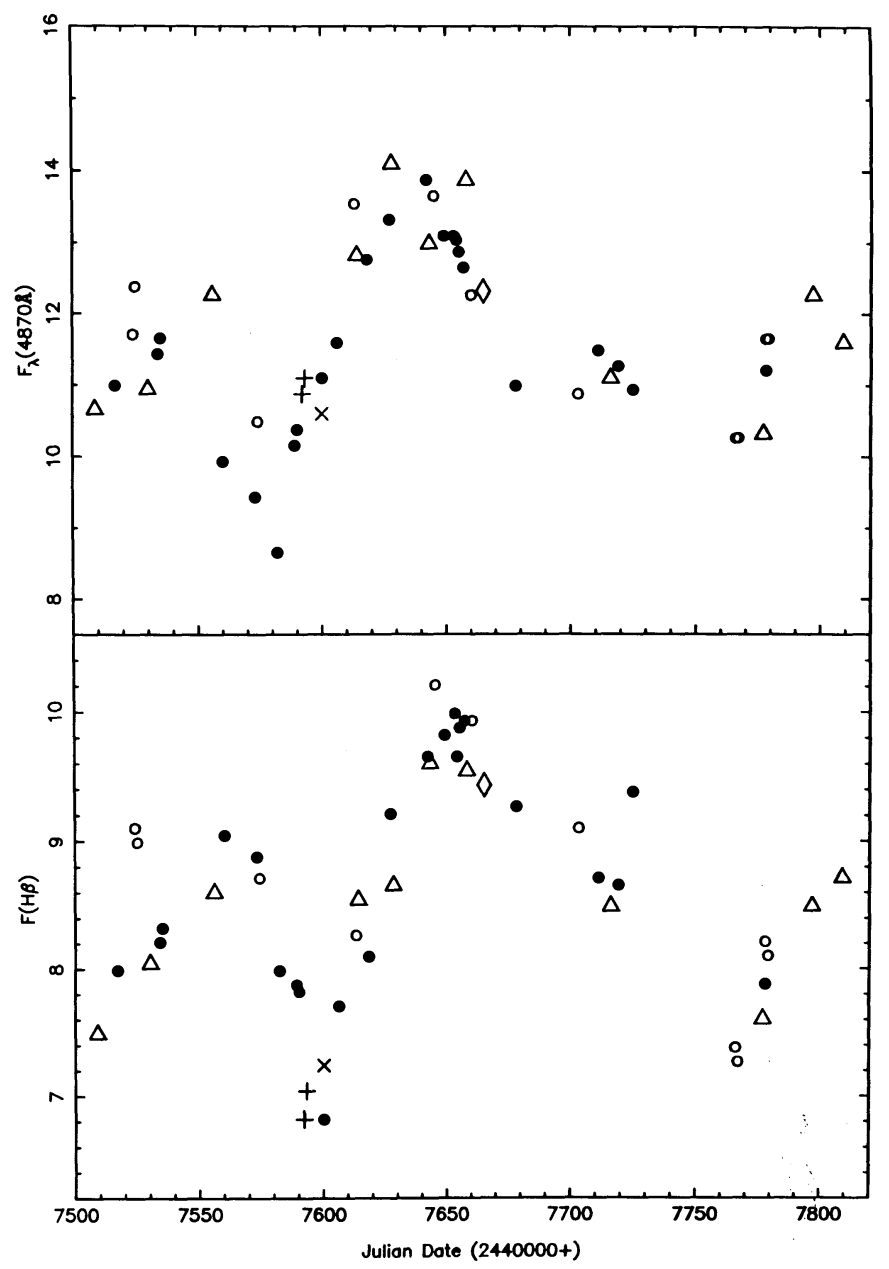

Fig. 2.-Continuum (upper panel) and $\mathrm{H} \beta$ (lower panel) flux measurements from high-quality spectra with similar entrance apertures. These measurements, from Table 6, are uncorrected for any aperture effects and put on an absolute flux scale by multiplying the measured flux ratios by the absolute [O III] $\lambda 5007$ flux given in Table 5. Filled circles: set A; diamonds: set G; triangles: set $\mathrm{H} 1$; open circles: set I; plus signs: set J; crosses: set K1.

that any differences between them reflect differences in the amount of [O III] $\lambda 5007$ measured, then we can compute a point-source correction factor $\varphi$ which is defined by the equation

$$
F(\mathrm{H} \beta)=\varphi F_{5007}\left(\frac{F(\mathrm{H} \beta)}{F([\mathrm{O} \mathrm{III}] \lambda 5007)}\right)_{\mathrm{obs}},
$$

where $F_{5007}$ is the adopted mean [O III] $\lambda 5007$ flux from Table 5 and the observed ratio is as given in Table 6 . We then compute a correction for the starlight for different apertures, which enters as an additive term $G$, i.e.,

$$
F_{\lambda}(4870 \AA)=\varphi F_{5007}\left(\frac{F_{\lambda}(4870 \AA)}{F([\mathrm{O} \text { III }] \lambda 5007)}\right)_{\mathrm{obs}}-G .
$$

By comparing pairs of simultaneous observations from different data sets, we can determine the constants $\varphi$ and $G$ which are needed to adjust the emission-line and continuum fluxes to a common scale. Furthermore, the formal uncertainties in $\varphi$ and $G$ reflect the uncertainties in the individual data sets, so we can determine the nominal uncertainties for each data set if we assume that the errors add in quadrature. 
In practice, we find that it is neither necessary nor desirable to base the intercalibration of different data sets only on observations that were made on the same day. If we require such strict simultaneity, we find that some data sets cannot be used at all because they have no points in common with other sets, and in other cases the intercalibration is very poorly determined because it is based on such a small number of points. If instead we base the comparison on pairs of points which are separated by 2 days or less, we find (a) that all of the data in Table 6 can be intercalibrated and $(b)$ that the number of pairs contributing to each comparison is greatly increased, thus improving the accuracy of the result. The fundamental assumption is that no significant line or continuum variability occurs on time scales shorter than $\sim 2$ days. The justification for this assumption is (1) that the homogeneous IUE data from Paper I show no evidence for significant short-term variations and (2) that relaxing the simultaneity requirement does not significantly change the formal uncertainties in the determination of either $\varphi$ or $G$, which is what would happen if there were real variability on short time scales. With regard to the latter point, evidence for real variability begins to appear on time scales longer than about 5 days. By regarding all data obtained over any 2 day interval as simultaneous, we effectively degrade our highest achievable temporal resolution. We believe, however, that the gain in temporal coverage and accuracy offsets this disadvantage.

Operationally, the intercalibration procedure is carried out by comparing some of the larger data sets, and then gradually building up the calibrated base by including additional data sets. All the data are calibrated relative to data set $\mathrm{A}$ because these data are fairly numerous, overlap reasonably well with most of the other data sets, and were obtained through a reasonably large aperture $\left(5^{\prime \prime} \times 77^{\prime \prime} 6\right)$. The fractional uncertainties in the continuum $\left[\sigma_{\text {cont }} / F_{\lambda}(4870 \AA) \approx 0.040\right]$ and the $\mathrm{H} \beta$ flux $\left[\sigma_{\text {line }} / F(\mathrm{H} \beta) \approx 0.035\right]$ for the similar data in Figure 2, which are all high-quality spectra obtained through large apertures, were determined by comparing all pairs of measurements separated by 2 days or less. It was also possible to assess independently the uncertainties in certain other data sets. The data from the $1.6 \mathrm{~m}$ Mount Hopkins telescope (set F) were sufficiently well sampled on short time scales to assess their internal accuracy reliably, and the fractional uncertainties in the continuum and the line are the same as for the data shown in Figure 2. The uncertainty in the Wise Observatory (set B) measurements $\left(\sigma_{\text {cont }} / F_{\lambda}(4870 \AA) \approx 0.040 ; \sigma_{\text {line }} / F(\mathrm{H} \beta) \approx 0.050\right)$ are taken from Netzer et al. (1990), and the uncertainty in the single Ohio State IDS (set G) data point $(5 \%$ in each parameter) is adopted from Peterson et al. (1990). No correction is applied to the latter point because of an insufficient number of nearly contemporaneous observations. For most of the other data sets, it was possible to estimate the mean uncertainties in the measurements by comparing them with measurements from other sets for which the uncertainties are known and by assuming that the uncertainties for each set add in quadrature. This procedure did not work well for some of the small data sets, and in such cases we simply adopted uncertainties from the larger data sets which were most similar in terms of spectrograph entrance aperture, spectral resolution, and signal-to-noise ratio. We note that this procedure seems to give fairly consistent error estimates and calibration constants regardless of the specific order in which the data bases are combined.

The intercalibration constants we use for each data set are
TABLE 7

FLUX SCALE Factors

\begin{tabular}{|c|c|c|}
\hline $\begin{array}{c}\text { Data } \\
\text { Set } \\
\text { (1) }\end{array}$ & $\begin{array}{c}\text { Point-Source } \\
\text { Scale Factor } \\
\varphi \\
(2)\end{array}$ & $\begin{array}{c}\text { Extended Source } \\
\text { Correction } G \\
\left(10^{-15} \mathrm{ergs} \mathrm{s}^{-1} \mathrm{~cm}^{-2} \AA^{-1}\right) \\
(3)\end{array}$ \\
\hline A & 1.000 & 0.000 \\
\hline В $\ldots \ldots \ldots \ldots \ldots$ & $1.008 \pm 0.043$ & $5.845 \pm 0.695$ \\
\hline C & $0.978 \pm 0.103$ & $-0.525 \pm 0.582$ \\
\hline $\mathrm{D}, \ldots \ldots \ldots \ldots \ldots$ & $0.902 \pm 0.035$ & $-3.016 \pm 0.491$ \\
\hline ..... & $0.988 \pm 0.044$ & $-0.048 \pm 0.661$ \\
\hline ........ & $0.931 \pm 0.045$ & $-2.349 \pm 0.371$ \\
\hline G & 1.000 & 0.000 \\
\hline H1 & $1.000 \pm 0.051$ & $0.135 \pm 0.957$ \\
\hline H2 $\ldots \ldots \ldots \ldots$ & $0.952 \pm 0.053$ & $-1.449 \pm 1.084$ \\
\hline I . . . . . . . . . . & $0.955 \pm 0.042$ & $0.373 \pm 1.024$ \\
\hline $\mathbf{J} \ldots \ldots \ldots \ldots$ & $1.112 \pm 0.052$ & $1.896 \pm 0.382$ \\
\hline K1 $1 \ldots \ldots \ldots \ldots$ & $1.013 \pm 0.053$ & $-0.446 \pm 0.116$ \\
\hline$\ldots$. & $0.983 \pm 0.048$ & $-1.143 \pm 1.249$ \\
\hline .......... & $0.926 \pm 0.060$ & $-1.682 \pm 0.720$ \\
\hline $\mathbf{M}$ & $1.040 \pm 0.066$ & $-0.748 \pm 0.799$ \\
\hline N1. & $0.888 \pm 0.064$ & $1.443 \pm 1.105$ \\
\hline N2 $\ldots \ldots \ldots \ldots$ & $0.960 \pm 0.055$ & $1.122 \pm 0.931$ \\
\hline ........ & $1.080 \pm 0.024$ & $-1.455 \pm 0.384$ \\
\hline …....... & $1.154 \pm 0.097$ & $2.551 \pm 0.889$ \\
\hline $\mathrm{Q} \ldots \ldots \ldots \ldots \ldots$ & $1.027 \pm 0.052$ & $-1.712 \pm 0.687$ \\
\hline
\end{tabular}

given in Table 7. It is important to note that (1) the pointsource correction factor is always close to unity, indicating that the ratio of point-source to narrow-line flux does not vary greatly with aperture (i.e., the narrow-line surface brightness distribution is in fact rather concentrated) and (2) the extended source (starlight) correction is generally in the expected sense, i.e., positive for much larger apertures than $5^{\prime \prime} \times 7^{\prime \prime} .6$ and negative for much smaller apertures. For apertures of similar size the correction factors have only a small effect.

The continuum and line measurements from Table 6 are adjusted to a common scale corresponding to measurements through a $5^{\prime \prime} \times 7^{\prime \prime} .6$ spectrograph entrance aperture by using the constants of Table 7 in equations (1) and (2). The resultant values of the continuum flux $F_{\lambda}(4870 \AA)$ and the line flux $F(\mathbf{H} \beta)$ are given in Table 8. A final light curve is produced by computing the variance-weighted average of all the measurements obtained on a given Julian Date; this light curve is given in Table 9 and shown in Figure 3. Also shown for comparison in Figure 3 are the $B$-band photometric measurements from Tables 2, 3, and 4; we have placed these measurements on an approximate flux scale by using the relationship

$$
B=-2.5 \log F_{v}-48.60
$$

(Oke and Gunn 1983), where $F_{v}$ is the flux in units of ergs s ${ }^{-1}$ $\mathrm{cm}^{-2} \mathrm{~Hz}^{-1}$.

\section{VARIABILITY ANALYSIS}

\section{a) Characteristics of the Data Base}

Table 8 gives 177 separate measurements of the optical continuum and $\mathrm{H} \beta$ fluxes in NGC 5548 between 1988 December 14 and 1989 October 10, a period of 301 days. By averaging measurements obtained on a given Julian Date, we produce a final light curve covering 129 independent epochs, as given in Table 9. For the purpose of time-series analysis, we exclude the final two points where the light curve is poorly sampled, which leaves 127 measurements over a 271 day period. The average interval between these observations is 3.1 days, and the median 
TABLE 8

SCAled Flux Measurements

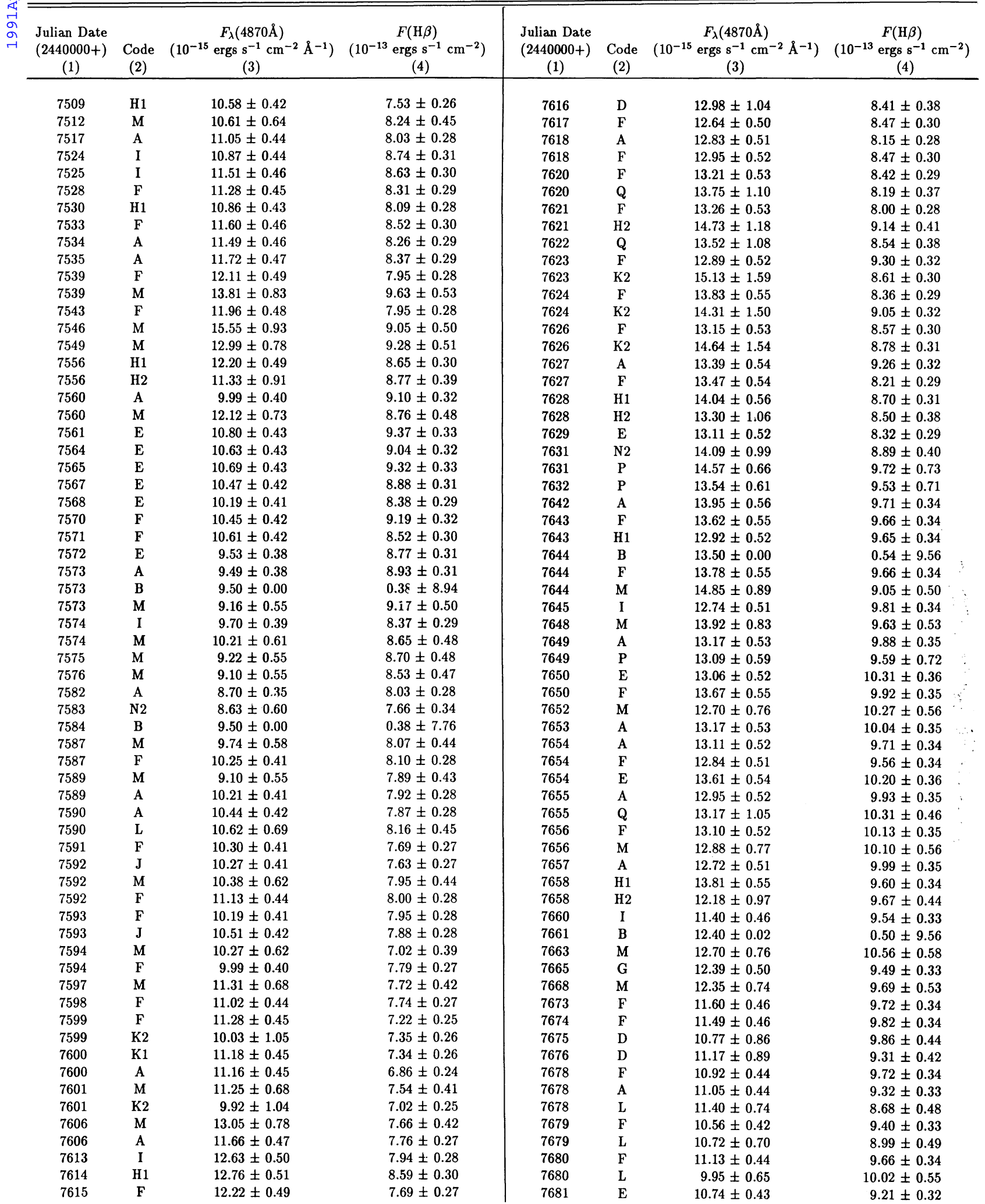


TABLE 8-Continued

\begin{tabular}{|c|c|c|c|}
\hline $\begin{array}{c}\text { Julian Date } \\
(2440000+) \\
(1)\end{array}$ & $\begin{array}{c}\text { Code } \\
(2)\end{array}$ & $\begin{array}{c}F_{\lambda}(4870 \AA) \\
\left(10^{-15} \mathrm{ergs} \mathrm{s}^{-1} \mathrm{~cm}^{-2} \AA^{-1}\right) \\
(3)\end{array}$ & $\begin{array}{c}F(\mathrm{H} \beta) \\
\left(10^{-13} \mathrm{ergs} \mathrm{s}^{-1} \mathrm{~cm}^{-2}\right) \\
(4)\end{array}$ \\
\hline 7681 & $\mathrm{~F}$ & $10.97 \pm 0.44$ & $9.35 \pm 0.33$ \\
\hline 7682 & $\mathrm{E}$ & $11.74 \pm 0.47$ & $9.21 \pm 0.32$ \\
\hline 7682 & $\mathrm{~F}$ & $11.18 \pm 0.45$ & $8.88 \pm 0.31$ \\
\hline 7683 & $\mathrm{~F}$ & $11.49 \pm 0.46$ & $9.45 \pm 0.33$ \\
\hline 7684 & $\mathrm{~F}$ & $10.66 \pm 0.43$ & $9.09 \pm 0.32$ \\
\hline 7685 & $\mathrm{~F}$ & $11.28 \pm 0.45$ & $8.99 \pm 0.31$ \\
\hline 7686 & $\mathrm{~F}$ & $10.45 \pm 0.42$ & $9.25 \pm 0.32$ \\
\hline 7687 & $\mathrm{C}$ & $10.57 \pm 0.85$ & $8.40 \pm 0.38$ \\
\hline 7699 & $\mathrm{~N} 1$ & $11.19 \pm 1.06$ & $7.68 \pm 0.46$ \\
\hline 7700 & N1 & $11.49 \pm 1.09$ & $7.68 \pm 0.46$ \\
\hline 7701 & $\mathrm{~N} 1$ & $11.64 \pm 1.11$ & $7.78 \pm 0.47$ \\
\hline 7702 & $\mathrm{~K} 2$ & $9.97 \pm 1.05$ & $8.34 \pm 0.29$ \\
\hline 7703 & $\mathrm{~K} 2$ & $9.37 \pm 0.98$ & $8.72 \pm 0.31$ \\
\hline 7703 & I & $10.07 \pm 0.40$ & $8.74 \pm 0.31$ \\
\hline 7705 & $\mathrm{~F}$ & $10.04 \pm 0.40$ & $8.57 \pm 0.30$ \\
\hline 7707 & $\mathrm{~F}$ & $11.08 \pm 0.44$ & $8.52 \pm 0.30$ \\
\hline 7708 & $\mathrm{~F}$ & $11.02 \pm 0.44$ & $8.57 \pm 0.30$ \\
\hline 7.709 & $\mathrm{~F}$ & $11.08 \pm 0.44$ & $8.52 \pm 0.30$ \\
\hline 7710 & $\mathrm{~F}$ & $10.92 \pm 0.44$ & $8.26 \pm 0.29$ \\
\hline 7710 & 0 & $11.28 \pm 0.90$ & $8.50 \pm 0.38$ \\
\hline 7711 & $\mathbf{A}$ & $11.55 \pm 0.46$ & $8.76 \pm 0.31$ \\
\hline 7711 & $\mathrm{~L}$ & $11.81 \pm 0.77$ & $8.37 \pm 0.46$ \\
\hline 7713 & $M$ & $11.08 \pm 0.67$ & $7.83 \pm 0.43$ \\
\hline 7715 & $\mathrm{~F}$ & $10.51 \pm 0.42$ & $8.21 \pm 0.29$ \\
\hline 7716 & $\mathrm{H} 1$ & $11.02 \pm 0.44$ & $8.54 \pm 0.30$ \\
\hline 7719 & $\mathbf{A}$ & $11.33 \pm 0.45$ & $8.70 \pm 0.31$ \\
\hline 7725 & A & $10.99 \pm 0.44$ & $9.43 \pm 0.33$ \\
\hline 7728 & N1 & $9.90 \pm 0.94$ & $7.93 \pm 0.48$ \\
\hline 7730 & N1 & $10.80 \pm 1.03$ & $7.68 \pm 0.46$ \\
\hline 7736 & $\mathrm{H} 2$ & $8.09 \pm 0.65$ & $8.18 \pm 0.37$ \\
\hline 7741 & $\mathrm{~L}$ & $7.37 \pm 0.48$ & $8.01 \pm 0.44$ \\
\hline 7742 & $\mathrm{C}$ & $7.29 \pm 0.58$ & $8.35 \pm 0.38$ \\
\hline 7748 & $\mathrm{C}$ & $8.06 \pm 0.64$ & $8.68 \pm 0.39$ \\
\hline 7749 & M & $7.36 \pm 0.44$ & $7.54 \pm 0.41$ \\
\hline 7754 & M & $7.13 \pm 0.43$ & $7.60 \pm 0.42$ \\
\hline 7757 & M & $8.81 \pm 0.53$ & $7.37 \pm 0.41$ \\
\hline 7758 & M & $10.50 \pm 0.63$ & $7.60 \pm 0.42$ \\
\hline 7759 & M & $9.57 \pm 0.57$ & $6.73 \pm 0.37$ \\
\hline 7765 & M & $7.83 \pm 0.47$ & $6.73 \pm 0.37$ \\
\hline 7766 & N1 & $8.52 \pm 0.81$ & $7.14 \pm 0.43$ \\
\hline 7766 & I & $9.48 \pm 0.38$ & $7.09 \pm 0.25$ \\
\hline 7767 & M & $8.81 \pm 0.53$ & $6.85 \pm 0.38$ \\
\hline 7767 & I & $9.48 \pm 0.38$ & $6.98 \pm 0.24$ \\
\hline 7777 & $\mathrm{H} 1$ & $10.24 \pm 0.41$ & $7.64 \pm 0.27$ \\
\hline 7778 & I & $10.82 \pm 0.43$ & $7.89 \pm 0.28$ \\
\hline 7778 & $\mathrm{~A}$ & $11.27 \pm 0.45$ & $7.92 \pm 0.28$ \\
\hline 7779 & I & $10.82 \pm 0.43$ & $7.78 \pm 0.27$ \\
\hline 7797 & $\mathrm{H} 1$ & $12.20 \pm 0.49$ & $8.54 \pm 0.30$ \\
\hline 7809 & $\mathrm{H} 1$ & $11.53 \pm 0.46$ & $8.76 \pm 0.31$ \\
\hline
\end{tabular}

interval is 1 day. The largest gaps in the coverage are 12 days (JD 2,447,687-JD 2,447,699) and 10 days (JD 2,447,632-JD $2,447,642$ and JD 2,447,767-JD 2,447,777), and there are no other gaps longer than 7 days.

We can use the data in Table 9 to perform a final check on our error estimates by examining the ratios of all pairs of measurements separated by 2 days or less. In Table 9 there are 144 independent pairs of measurements within 2 days of one another. The dispersion about the mean (unity), divided by $2^{1 / 2}$, provides an estimate of the typical uncertainty in a single measurement. For the continuum, we find that the mean fractional error in a given measurement is 0.040 . The average fractional uncertainty, from the quoted estimates for these same 144 measurements in Table 9, is 0.045 . This implies that our error estimates are probably quite good, and perhaps overesti-

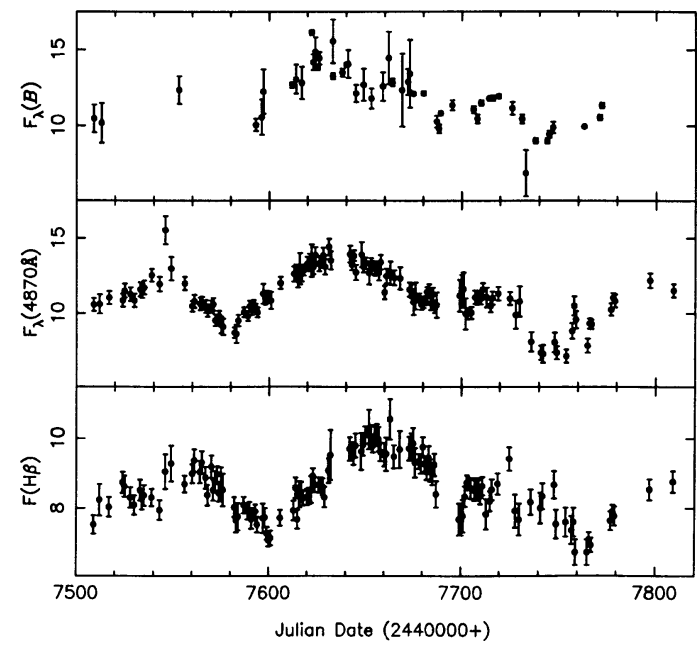

FIG. 3.-Top panel: photometric B-magnitudes from Tables 2, 3, and 4 placed on an approximate linear flux scale by using eq. (3). Middle panel: continuum fluxes at $4870 \AA$ from the optical spectra, as given in Table 9 . The continuum fluxes are in units of $10^{-15} \mathrm{ergs} \mathrm{s}^{-1} \mathrm{~cm}^{-2} \AA^{-1}$. Bottom panel: $\mathrm{H} \beta$ fluxes from Table 9 , in units of $10^{-13} \mathrm{ergs} \mathrm{s}^{-1} \mathrm{~cm}^{-2}$.

mated on average by about $10 \%$. Similarly, examination of the $\mathrm{H} \beta$ emission-line fluxes indicates that the fractional uncertainty is 0.033 , compared with the value of 0.037 computed from the Table 9 entries. Again, this indicates that our errors may be very slightly overestimated.

Inspection of Figure 3 suggests that the continuum measurement on JD $2,447,546$ is anomalously high. This spectrum has been carefully inspected and remeasured, and the original data were re-reduced in an attempt to determine whether or not there might indeed be a problem with these data. We were unable to find anything obviously wrong in the original data or with the data reduction, and we have therefore kept this measurement in the data base despite our suspicion that it is too high.

\section{b) Variability Amplitude}

Significant variability was detected in both the continuum and the $\mathrm{H} \beta$ emission line. This is evident from inspection of the high-state and low-state spectra shown in Figure 4. The con-

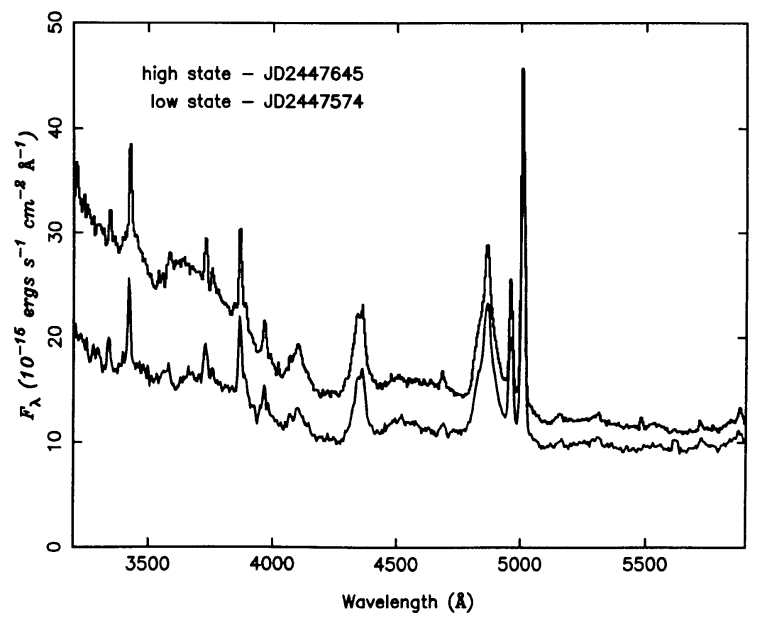

FIG. 4.- - High-state and low-state optical spectra of NGC 5548. The two spectra shown here were obtained with the $2.3 \mathrm{~m}$ Steward Observatory telescope on Kitt Peak. 
TABLE 9

Optical Continuum and h $\beta$ Light Curves

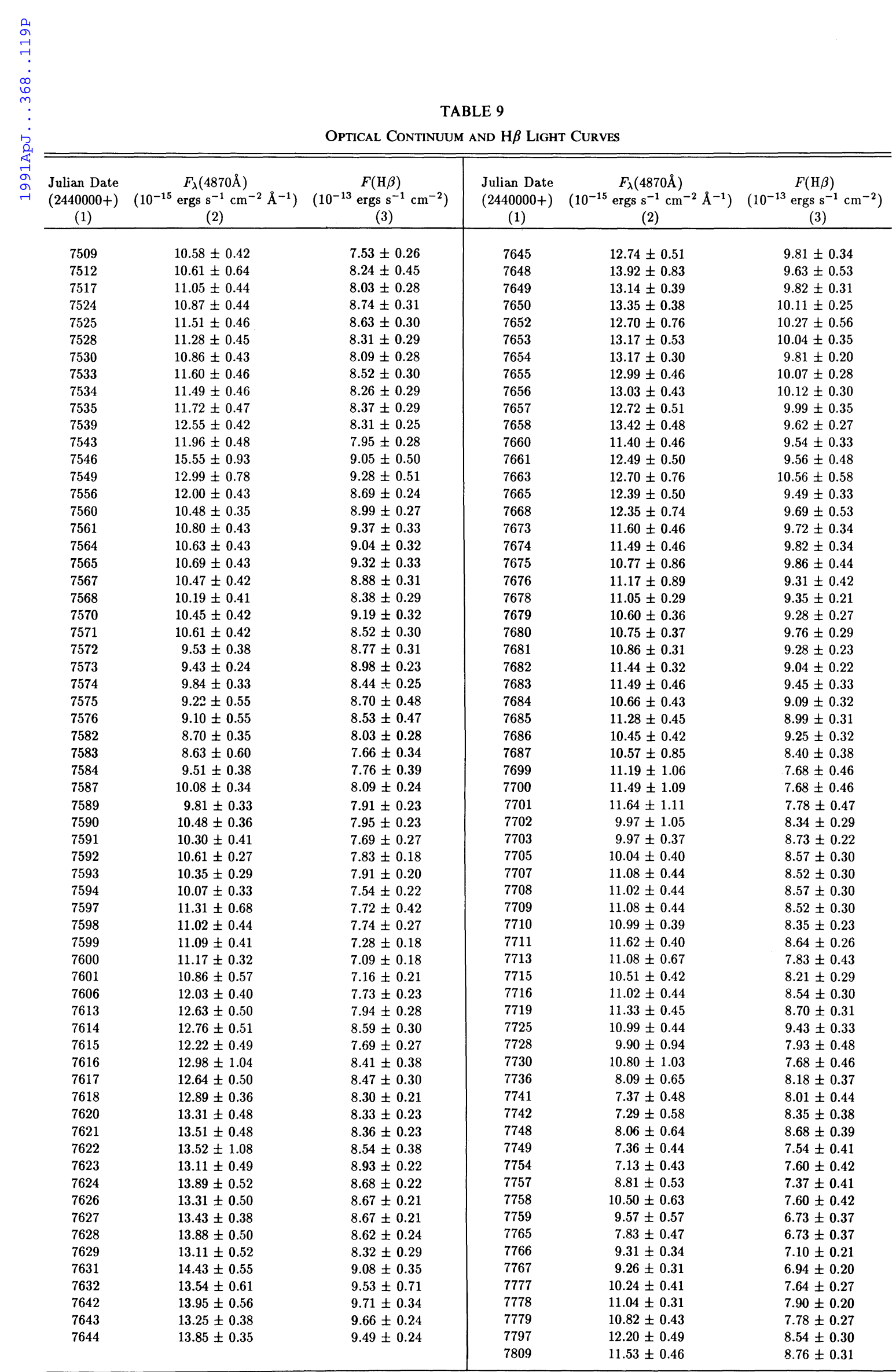


TABLE 10

VARIABILITY PARAMETERS

\begin{tabular}{|c|c|c|c|}
\hline $\begin{array}{c}\text { Feature } \\
\text { (1) }\end{array}$ & $\begin{array}{c}\text { Mean } \\
\text { Flux }^{\mathbf{a}} \\
(2)\end{array}$ & $\begin{array}{c}F_{\text {var }} \\
(3)\end{array}$ & $\begin{array}{c}R_{\max } \\
\text { (4) }\end{array}$ \\
\hline$F_{\lambda}(1350 \AA)^{\mathrm{b}}$ & 43.9 & 0.32 & 4.64 \\
\hline$F_{\lambda}(4870 \AA) \quad \ldots \ldots \ldots \ldots$ & 11.3 & 0.14 & 2.18 \\
\hline $\mathrm{Ly} \alpha+\mathrm{N} v \lambda 1240^{\mathrm{b}} \ldots$. & 76.0 & 0.17 & 2.14 \\
\hline $\mathbf{H} \beta \ldots \ldots \ldots \ldots \ldots$ & 8.59 & 0.093 & 1.57 \\
\hline
\end{tabular}

anits as in Table 9.

b From Paper I (GEX values).

tinuum shows the same pattern of variability as seen in the $I U E$ data presented in Paper $\mathrm{I}$, and inspection of Figure 3 shows that $\mathrm{H} \beta$, like the ultraviolet emission lines studied in Paper I, also varies in the same pattern but with a short delay. The similarity of the $B$-band measurements, the IUE continuum measurements (ultraviolet fluxes and broad-band optical fluxes as measured with the fine-error sensor), and the continuum measurements based on the optical spectra reassures us that our flux calibration is robust.

In Table 10 we present parameters that characterize the amplitude of the optical continuum and $\mathrm{H} \beta$ variability. The fractional variation $F_{\text {var }}$ is, as defined in Paper I, the ratio of the rms fluctuation to the mean flux, and is corrected for the effect of measurement errors. The parameter $R_{\max }$ is the ratio of maximum to minimum flux. Note that both of these parameters are subject to strong systematic effects. While accurate determination of the stellar continuum is very difficult and will be discussed elsewhere, we can make the approximation that at minimum light, about half of the continuum emission through our nominal $5^{\prime \prime} \times 7 " .6$ aperture is due to a constant starlight contribution from the host galaxy, and accounting for this will about double the quoted value of $F_{\text {var }}$ and increase $R_{\max }$ as well. Similarly, the amplitude of variation of $\mathrm{H} \beta$ will increase when the constant narrow-line component is taken into account, although this is also true for the ultraviolet lines.

\section{c) Time-Series Analysis}

Inspection of Figure 3 shows the principal result of this study, namely, that the broad $\mathrm{H} \beta$ emission feature varies in the same fashion as the continuum, but with a short delay. These data are replotted in Figure 5, which also shows for comparison purposes the SIPS measurements for the ultraviolet continuum at $1350 \AA$ and the Ly $\alpha$ line (see Paper I for the details of how the ultraviolet measurements were made). Comparison of the light curves for the two emission lines reveals the very important result that they do not vary strictly in phase.

We can quantify the phase shift or "lag" between different light curves by computing their mutual cross-correlations. When the data are unevenly sampled, as they are here, special techniques must be used for these computations. Here we will employ two methods which are commonly used in the context of AGN emission-line variability: (1) the interpolation method of Gaskell and Sparke (1986), with the particular implementation described by Gaskell and Peterson (1987), and (2) the discrete correlation function of Edelson and Krolik (1988). The sampling in this data set is so dense relative to the dominant fluctuation time scales that these two methods give results that are in close agreement.

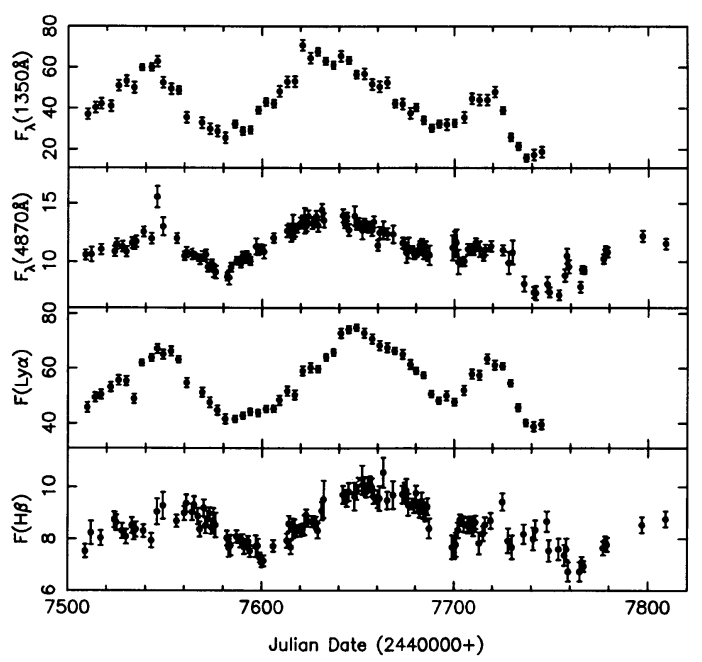

FIG. 5.-Light curves are shown for the ultraviolet (1350 $\AA$ ) and optical $(4870 \AA)$ continua and the $\operatorname{Ly} \alpha$ and $\mathrm{H} \beta$ emission lines. The continuum fluxes are in units of $10^{-15} \mathrm{ergs} \mathrm{s}^{-1} \mathrm{~cm}^{-2} \AA^{-1}$, and the emission-line fluxes are in units of $10^{-13} \mathrm{ergs} \mathrm{s}^{-1} \mathrm{~cm}^{-2}$.

i) Comparison of the Ultraviolet and Optical Continua

We first investigate the possibility of a phase shift between the ultraviolet and optical continuum by cross-correlating the ultraviolet $1350 \AA$ SIPS light curve from Paper I with the optical continuum light curve presented in Table 9. The result is shown graphically in Figure 6. The peak in the crosscorrelation function (CCF) formally occurs at +2 days, in the sense that the optical continuum lags behind the ultraviolet continuum. However, this lag is half the interval between the $I U E$ observations, and comparable to the temporal resolution of the optical data, and therefore is probably not significant. We also note that no lag is found between the ultraviolet continuum measurements and the photometric measurements shown in the top panel of Figure 3.

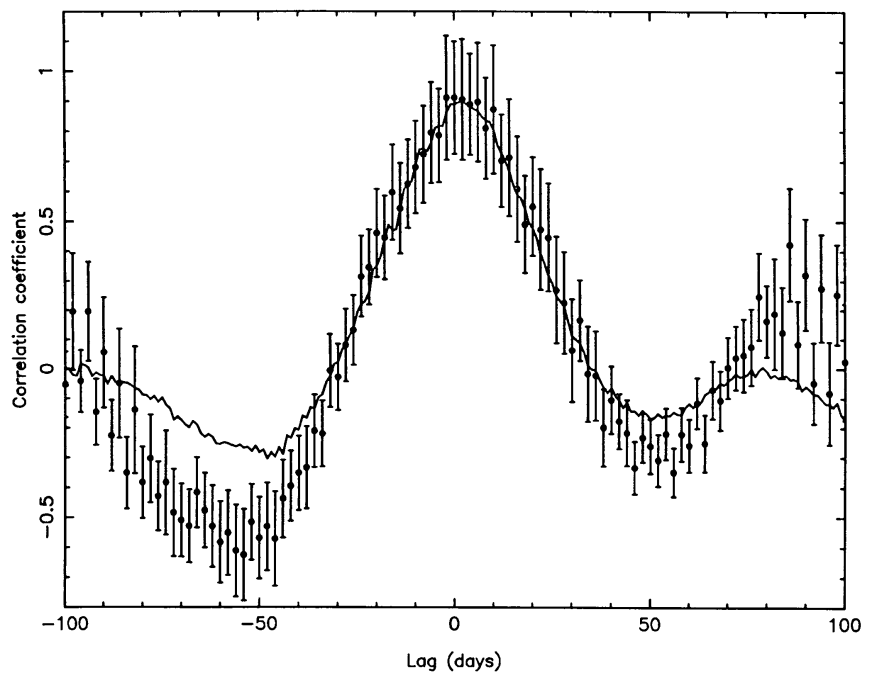

Fig. 6.-Interpolation cross-correlation function, shown as a smooth line, and the discrete correlation function (DCF), shown as individual points with associated uncertainties, for the ultraviolet continuum $(1350 \AA)$ and the optical continuum ( $4870 \AA)$. The optical continuum lags the ultraviolet continuum by $\sim 2$ days, which is less than the temporal resolution of this experiment and therefore not significant. 


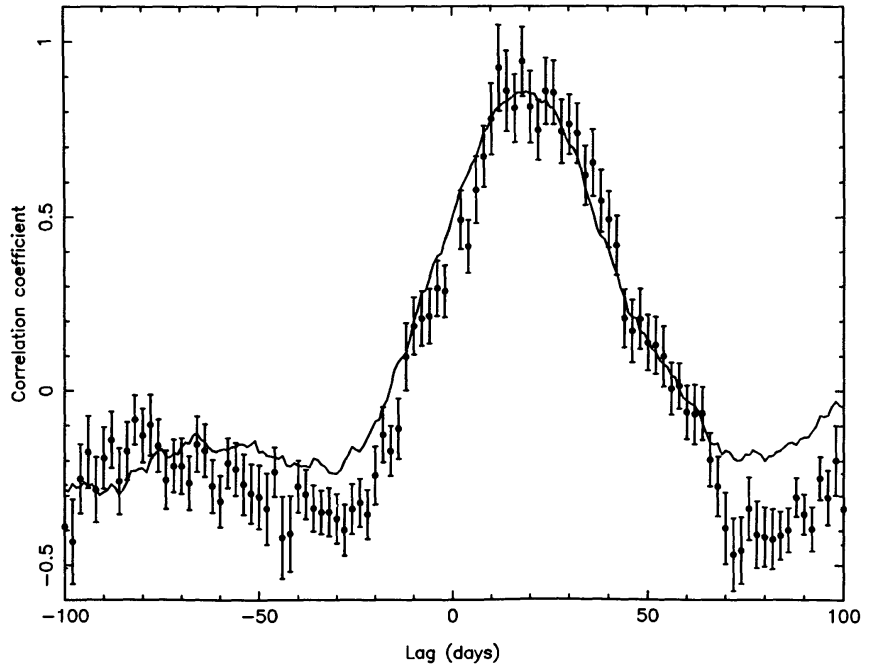

FIG. 7.-Interpolation cross-correlation function and DCF, plotted as in Fig. 6, for the optical continuum and $\mathrm{H} \beta$. The bin width for the DCF is 2 days, since the intercalibration process smooths out variations on shorter time scales. The $\mathrm{H} \beta$ emission line lags the optical continuum by about 20 days. The cross-correlation function is broad and flat-topped, and this is attributable to the width of the continuum autocorrelation function, shown in Fig. 8.

\section{ii) Emission-Line Results}

The lag between the optical continuum and $\mathrm{H} \beta$ measurements is found to be $\sim 20$ days, as seen in Figure 7 and Table 11. This result should be compared with the computed lags for other emission lines as given in Paper I. We note in particular that the lag between the ultraviolet continuum and Ly $\alpha$ is found to be $\sim 10$ days. The difference between the response of $\mathrm{Ly} \alpha$ and that of $\mathrm{H} \beta$ can be substantiated further by a direct cross-correlation of the light curves of these emission lines, from which we find that $\mathbf{H} \beta$ lags $\operatorname{Ly} \alpha$ by $\sim 8$ days. This is consistent with the above results and cross-correlation uncertainties of a few days. The cross-correlation functions for Ly $\alpha$ and $\mathrm{H} \beta$ are shown in Figure 8 , along with the autocorrelation function (ACF) for the optical continuum. The CCF is the convolution of the transfer function for the BLR and the ACF (see Penston 1990). The similar widths of the CCF and ACF imply that the transfer function itself must be rather narrow compared with the ACF.

\section{iii) Comments on Uncertainties}

As noted in Paper I, there is no generally accepted method for assigning uncertainties to cross-correlation lags. Moreover, interpreting both the lag and its uncertainty requires knowing

TABLE 11

Cross-Correlation Results

\begin{tabular}{llcc}
\hline \hline $\begin{array}{c}\text { Parameter } \\
(1)\end{array}$ & \multicolumn{1}{c}{$\begin{array}{c}\text { Parameter } \\
(2)\end{array}$} & $\begin{array}{c}\Delta t_{\text {peak }} \\
(\text { days }) \\
(3)\end{array}$ & $\begin{array}{c}r_{\max } \\
(4)\end{array}$ \\
\hline$F_{\lambda}(1350 \AA)$ & $F_{\lambda}(4870 \AA)$ & 2 & 0.91 \\
$F(\mathrm{FES})$ & $F_{\lambda}(4870 \AA)$ & 2 & 0.84 \\
$F_{\lambda}(1350 \AA)$ & $F_{\lambda}(B)$ & 0 & 0.87 \\
$F_{\lambda}(1350 \AA)$ & $F(\mathbf{L y} \alpha)$ & 10 & 0.88 \\
$F_{\lambda}(1350 \AA)$ & $F(\mathbf{H} \beta)$ & 21 & 0.86 \\
$F_{\lambda}(4870 \AA)$ & $F(\mathbf{H} \beta)$ & 19 & 0.86 \\
$F_{\lambda}(4870 \AA)^{\mathrm{a}}$ & $F(\mathbf{H} \beta)^{\mathrm{a}}$ & 20 & 0.86 \\
$F(\mathrm{Ly} \alpha)$ & $F(\mathbf{H} \beta)$ & 8 & 0.84 \\
\hline
\end{tabular}

a Subset from Fig. 2.

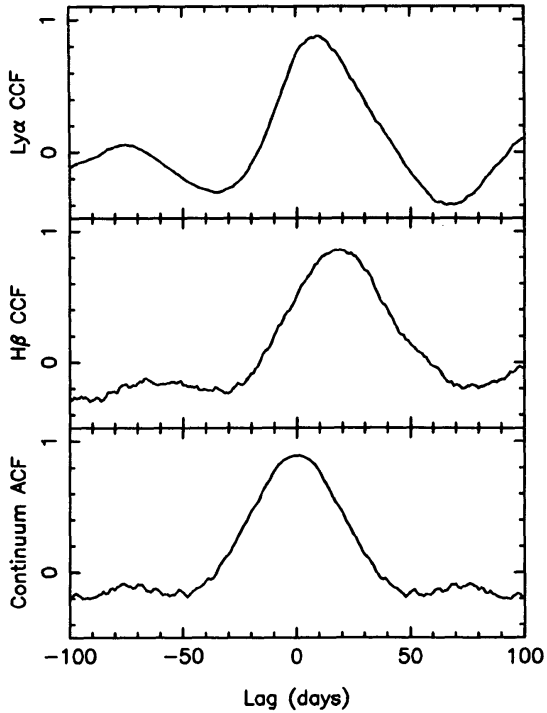

FIG. 8.-Top panel: ultraviolet continuum-Ly $\alpha$ cross-correlation function; middle panel: optical continuum- $\mathrm{H} \beta$ cross-correlation function. The optical continuum autocorrelation function is shown for comparison in the bottom panel.

or assuming the BLR geometry and orientation. Therefore, we will only note the results of computing the uncertainties from a variety of methods; the important point is that each of these methods indicates that the uncertainty in the lag is small, no worse than a few days. For the sake of simplicity and definiteness, all computations will assume a thin spherical BLR centered on the continuum source, even though this particular geometry is highly unlikely. The sole virtue of this geometry is its simplicity; the transfer function for such a region is specified by a single parameter, the radius.

1. Gaskell and Peterson (1987) give a formula for the uncertainty in the lag, which depends on the width and maximum of the cross-correlation function and the total number of data points. For the cross-correlations summarized in Table 11, errors of $\epsilon \approx 2$ days are indicated by this formula.

2. Maoz and Netzer (1989) use the cross-correlation peak distribution (CCPD) from Monte Carlo simulations to assign an uncertainty to the lag. In our simulations, we start with the optical continuum points given in Table 9 and generate a model light curve by interpolating linearly between observations separated by more than one day and then smoothing this light curve. From this model continuum light curve, we construct a model emission-line light curve by convolving the continuum light curve with the transfer function for the thin spherical shell of radius $R$. We then resample the model light curves at 127 different points in such a way as to preserve the distribution of intervals between observations. Gaussiandistributed random observational errors are introduced into each of the data points drawn from the model light curves, and the sampled data are then cross-correlated in the same way as real data to determine the location of the peak in the CCF. The CCPD is built up by repeating this process a large number of times for a given value of $R$. We consider different values of $R$ to find the largest value of $R$ for which a cross-correlation lag no larger than the observed value will be obtained at least a third of the time, and this is adopted as a $1 \sigma$ upper limit on the observed size. A similar procedure is followed to find the $1 \sigma$ lower limit. The result is $20 \pm 3$ days for the optical continuum- $\mathrm{H} \beta$ cross-correlation. 
3. Each point in the discrete correlation function (DCF) has an associated uncertainty which is derived from the uncertainties in the observed fluxes which contribute to the correlation at a given lag. It is thus possible to perform a meaningful fit to the DCF points near the peak of the function to locate the peak and to estimate the accuracy to which it is known. We have fitted a parabola to the DCF points with correlation amplitudes $r>0.6$, and we find that the DCF peak occurs at $21.4 \pm 3.0$ days for the optical continuum- $\mathrm{H} \beta$ crosscorrelation, at $2.1 \pm 0.4$ days for the ultraviolet continuumoptical continuum cross-correlation, and at $8.9 \pm 1.6$ days for the Ly $\alpha-\mathrm{H} \beta$ cross-correlation.

It is important to remember at this point that the peak in the CCF for an emission line gives very limited information about the actual geometry of the line-emitting region. More sophisticated techniques, which are beyond the scope of this paper, are required to solve the transfer equation, which contains the information on the BLR geometry. The small uncertainties which we derive for the various CCFs show only that the location of the peak in the CCF is a well-determined quantity. However well determined it is, the lag by itself does not necessarily provide a strong constraint on the distribution of lineemitting material. Moreover, the truly causal connection is with the unobservable ionizing continuum. Based on the crosscorrelation between the observed ultraviolet and optical continuum measurements, we believe it likely that the optical continuum tracks the ionizing continuum with a lag of no more than a few days.

\section{CONCLUSIONS}

An intensive international ground-based campaign to monitor the Seyfert 1 galaxy NGC 5548 has detected the same strong variations observed by the IUE satellite during the same period. The combined ultraviolet and optical effort has produced a data base of unprecedented size and quality for studies of AGN variability. On the basis of the initial analysis of these data, we can conclude the following:

1. The optical continuum shows the same qualitative behavior as the ultraviolet continuum. Cross-correlation of the continuum measurements in these two separate wavelength ranges reveals no statistically significant temporal shift between them.

2. The broad $\mathrm{H} \beta$ emission line varies in response to the observed continuum variations with a lag of $\sim 20$ days. It is particularly noteworthy that this lag is significantly longer than the observed lag for Ly $\alpha$, i.e., $\sim 10$ days. Direct crosscorrelation of the $\mathrm{Ly} \alpha$ and $\mathrm{H} \beta$ light curves shows a lag of $\sim 8$ days, which lends credence to this result.

We wish to acknowledge the support and encouragement of numerous members of the AGN community, many of whom were coauthors of Paper I, who made material contributions to the planning of the ground-based program and its $I U E$ counterpart. D. Alloin and J. Clavel in particular are thanked for their efforts. Much of the planning and organization of this project took place at the Segovia and Georgia State University conferences in the autumn of 1987, and at IAU Symposium 134 in Santa Cruz in the summer of 1988, and the important role played by the organizers and participants at these conferences is also acknowledged. We are very grateful to the Directors and Telescope Allocation Committees of our various observatories for their support of this demanding project. This project received special support by NASA through a grant administered by the American Astronomical Society, and individual investigators have benefited from support from a number of agencies, including the following: the National Science Foundation: AST-8702691 and AST-8915258 (Ohio State University, AST-8611457 (University of California, Santa Cruz), RII-8800600 and AST-9058510 (University of Arizona), AST-8821839 (University of Florida), AST-8714937 (University of Texas), AST-8957063 and AST-9003829 (University of California, Berkeley), AST-8614510 (University of California), and USE-8750955 (Colgate University under the College Science Instrumentation Program); NASA: NAG51366 (Ohio State University), NAS5-29293 (University of Arizona), and NAG5-1194 (Johns Hopkins University); DFG: Bi 191/G-2 and Ko 857/7-1,2 (Universitäts-Sternwarte Göttingen); the National Science and Engineering Research Council of Canada (Mount Royal College); the American Astronomical Society Small Grants Program (Colgate University); and the US-Israel Binational Science Foundation (grant $80 / 00085$ to Wise Observatory). For hospitality extended during part of this investigation, B. M. P. thanks the director and staff of Lick Observatory and M. V. P. acknowledges the Institute of Astronomy. The Isaac Newton telescope is run by the Royal Greenwich Observatory at the Spanish Observatorio del Roque de los Muchachos of the Instituto de Astrofísica de Canarias.

\section{REFERENCES}

Clavel, J., et al. 1991, Ap. J., in press (Paper I).

Edelson, R. A., and Krolik, J. H. 1988, Ap. J., 333, 646

Gaskell, C. M., and Peterson, B. M. 1987, Ap. J. Suppl., 65, 1.

Gaskell, C. M., and Sparke, L. S. 1986, Ap. J., 305, 175.

Maoz, D., and Netzer, H. 1989, M.N.R.A.S., 236, 21.

Maoz, D., et al. 1990, Ap. J., 351, 75.

Netzer, H. 1989, Comm. Ap., 14, 137.

Netzer, H., et al. 1990, Ap. J., 353, 108.

Oke, J. B., and Gunn, J. E. 1983, Ap. J., 266, 713.
Penston, M. J. Penston, M. V and Sandage, A. 1971, Pub. A.S.P. 83, 783.

Penston, M. V. 1990, in Variability of Active Galactic Nuclei, ed. H. R. Miller and P. J. Witta (Cambridge: Cambridge University Press), in press. Peterson, B. M. 1988, Pub. A.S.P., 100, 18.

Peterson, B. M., Reichert, G. A., Korista, K. T., and Wagner, R. M. 1990, Ap. J., 352, 68.

Stone, R. P. S. 1977, Ap. J., 218, 767.

Wilson, A. S., Wu, X., Heckman, T. M., Baldwin, J. A., and Balick, B. 1989, Ap. $J ., 339,729$.

T. J. Balonek and J. A. Christensen: Department of Physics and Astronomy, Colgate University, Hamilton, NY 13346

E. S. Barker, A. L. Cochran, S. R. Sawyer, B. J. Wills, and D. Wills: Department of Astronomy, University of Texas, RLM 15.308, Austin, TX 78712

J. Bechtold and P. S. Smith: Steward Observatory, University of Arizona, Tucson, AZ 85721

R. Bertram and R. M. WAGNER : Lowell Observatory, Mars Hill Road, 1400 West, Flagstaff, AZ 86001 
N. G. BochKarev: Sternberg State Astronomical Institute, University of Moscow, Universitetskij prosp. 13, Moscow V-234, USSR

M. J. Bolte: Lick Observatory, University of California at Santa Cruz, Santa Cruz, CA 95064

D. Bond, D. Crampton, J. B. Hutchings, L. SAdDlemyer, and P. F. Younger: Dominion Astrophysical Observatory, 5071 West Saanich Road, Victoria, BC, Canada V8X 4M6

T. A. Boroson: Kitt Peak National Observatory, National Optical Astronomy Observatories, P.O. Box 26732, Tucson, AZ 85726

M. T. Carini and H. R. Miller: Department of Physics and Astronomy, Georgia State University, Atlanta, GA 30303

T. E. CARONE: Space Sciences Laboratory, University of California at Berkeley, Berkeley, CA 94720

S. D. Clements, R. J. LeAcock, and A. G. Smith: Department of Astronomy, University of Florida, Gainesville, FL 32611

R. D. Cohen and E. I. Rosenblatt: Center for Astrophysics and Space Science, University of California at San Diego, C-011, La Jolla, CA 92093

M. DiEtrich, K. J. Fricke, and W. KollatschNY: Universitäts-Sternwarte Göttingen, Geismarlandstrasse 11, D-3400 Göttingen, Federal Republic of Germany

M. Elvis, J. P. Huchra, and B. J. Wilkes: Center for Astrophysics, 60 Garden Street, Cambridge, MA 02138

A. Ferguson and J. H. Krolik: Department of Physics and Astronomy, Johns Hopkins University, Homewood Campus, Baltimore, MD 21218

A. V. Filippenko, M. W. Richmond, and J. C. Shields: Department of Astronomy, University of California at Berkeley, Berkeley, CA 94720

C. M. Gaskell and G. M. MacAlpine: Department of Astronomy, University of Michigan, Dennison Building, Ann Arbor, MI 48109

J. P. HalPern: Department of Physics, Columbia University, 538 West 120th Street, New York, NY 10027

A. P. KoratKar: Space Telescope Science Institute, 3700 San Martin Drive, Baltimore, MD 21218

K. T. Korista, S. L. Morris, and R. J. Weymann: Observatories of the Carnegie Institution of Washington, 813 Santa Barbara Street, Pasadena, CA 91101

N. J. Lame, B. M. Peterson, and R. W. Pogge: Department of Astronomy, The Ohio State University, 174 West 18th Avenue, Columbus, $\mathrm{OH} \quad 43210$

A. LaOR and H. Netzer: School of Physics and Astronomy and the Wise Observatory, Tel-Aviv University, Ramat Aviv, Tel Aviv 69978 Israel

M. A. Malkan: Department of Astronomy, University of California at Los Angeles, Math-Science Building, Los Angeles, CA 90024

D. MAOz: Institute for Advanced Study, Princeton, NJ 08540

C. L. M. Oliveira: Department of Astronomy, University of British Columbia, Vancouver, BC, Canada V6T 1W5

J. PENFold: Department of Mathematics, Physics, and Engineering, Mount Royal College, Calgary, Alta., Canada T3E 6K6

M. V. Penston: Royal Greenwich Observatory, Madingley Road, Cambridge CB3 0EZ, United Kingdom

E. Pérez: Instituto de Astrofísica de Canarias, E-38200 La Laguna, Tenerife, Spain

W. Romanishin: Department of Physics and Astronomy, University of Oklahoma, Norman, OK 73019

A. SADuN: Department of Physics and Astronomy, Bradley Observatory, Agnes Scott College, Decatur, GA 30030

A. I. Shapovalova: Special Astrophysical Observatory, USSR Academy of Sciences, Nizhni Arkhys Stavropolsky Kraj, 357140 USSR 
H. A. Smiтh: Department of Astronomy, Michigan State University, East Lansing, MI 48824

W.-H. Sun: Institute of Physics and Astronomy, National Central University, 32054 Chung-li, Taiwan

U. THIELE: Max-Planck-Institut für Astronomie, Koenigstuhl, D-6900 Heidelberg, Germany

T. J. TURnER: Laboratory for High Energy Astrophysics, Goddard Space Flight Center, Greenbelt, MD 20771

S. VeILlEUX: Institute for Astronomy, University of Hawaii, 2680 Woodland Drive, Honolulu, HI 96822 